\title{
Correlates of Gross Motor Competence in Children and Adolescents: A Systematic Review and Meta-Analysis
}

\author{
Lisa M. Barnett ${ }^{1}$ - Samuel K. Lai ${ }^{1}$ - Sanne L. C. Veldman ${ }^{2}$ Louise L. Hardy ${ }^{3}$. \\ Dylan P. Cliff ${ }^{2} \cdot$ Philip J. Morgan $^{4} \cdot$ Avigdor Zask $^{5,6,7} \cdot$ David R. Lubans $^{4}$ • \\ Sarah P. Shultz ${ }^{8}$ - Nicola D. Ridgers ${ }^{9}$ Elaine Rush ${ }^{10}$ • Helen L. Brown' \\ Anthony D. Okely ${ }^{2}$
}

Published online: 19 February 2016

(c) The Author(s) 2016. This article is published with open access at Springerlink.com

\begin{abstract}
Background Gross motor competence confers health benefits, but levels in children and adolescents are low. While interventions can improve gross motor competence, it remains unclear which correlates should be targeted to ensure interventions are most effective, and for whom targeted and tailored interventions should be developed. Objective The aim of this systematic review was to identify the potential correlates of gross motor competence
\end{abstract}

Lisa M. Barnett

lisa.barnett@deakin.edu.au

1 Faculty of Health, School of Health and Social Development, Deakin University, Building BC, 221 Burwood Hwy, Burwood, Melbourne 3125, Australia

2 Faculty of Social Sciences, Early Start Research Institute, University of Wollongong, Wollongong, Australia

3 Prevention Research Collaboration, University of Sydney, Sydney, Australia

4 Faculty of Education and Arts, Priority Research Centre in Physical Activity and Nutrition, University of Newcastle, Newcastle, Australia

5 Northern NSW Health Promotion, Lismore, Australia

6 University Centre for Rural Health North Coast, School of Public Health, The University of Sydney, Sydney, Australia

7 School of Health and Human Sciences, Southern Cross University, Lismore, Australia

8 School of Sport and Exercise, Massey University, Wellington, New Zealand

9 Faculty of Health, Centre for Physical Activity and Nutrition Research, Deakin University, Melbourne, Australia

10 Centre for Child Health Research, Auckland University of Technology, Auckland, New Zealand in typically developing children and adolescents (aged 3-18 years) using an ecological approach.

Methods Motor competence was defined as gross motor skill competency, encompassing fundamental movement skills and motor coordination, but excluding motor fitness. Studies needed to assess a summary score of at least one aspect of motor competence (i.e., object control, locomotor, stability, or motor coordination). A structured electronic literature search was conducted in accordance with the Preferred Reporting Items for Systematic Reviews and Meta-Analyses statement. Six electronic databases (CINAHL Complete, ERIC, MEDLINE Complete, PsycINFO $^{\circledR}$, Scopus and SPORTDiscus with Full Text) were searched from 1994 to 5 August 2014. Meta-analyses were conducted to determine the relationship between potential correlates and motor competency if at least three individual studies investigated the same correlate and also reported standardized regression coefficients.

Results A total of 59 studies were identified from 22 different countries, published between 1995 and 2014. Studies reflected the full range of age groups. The most examined correlates were biological and demographic factors. Age (increasing) was a correlate of children's motor competence. Weight status (healthy), sex (male) and socioeconomic background (higher) were consistent correlates for certain aspects of motor competence only. Physical activity and sport participation constituted the majority of investigations in the behavioral attributes and skills category. Whilst we found physical activity to be a positive correlate of skill composite and motor coordination, we also found indeterminate evidence for physical activity being a correlate of object control or locomotor skill competence. Few studies investigated cognitive, emotional and psychological factors, cultural and social 
factors or physical environment factors as correlates of motor competence.

Conclusion This systematic review is the first that has investigated correlates of gross motor competence in children and adolescents. A strength is that we categorized correlates according to the specific ways motor competence has been defined and operationalized (object control, motor coordination, etc.), which enables us to have an understanding of what correlates assist what types of motor competence. Indeed our findings do suggest that evidence for some correlates differs according to how motor competence is operationalized.

\section{Key Points}

Increasing age was the most consistent correlate of all aspects of motor competence.

Aside from age, correlates of motor competence differ according to how motor competence is operationalized.

Investigating correlates of motor skills in children and adolescents is an emerging area with much scope for future investigation.

\section{Introduction}

Motor competence can be defined as a person's ability to execute different motor acts, including coordination of fine and gross motor skills that are necessary to manage everyday tasks [1]. Gross motor competence in particular plays an important role in growth, development and opportunities to lead an active lifestyle [2]. Gross motor competence is often specified as proficiency in a range of fundamental movement skills (e.g., throwing, catching, running) that are ideally learnt during the preschool and early school years [3-5]. These provide a foundation for children to develop more specialized movement sequences, such as sport-specific [6] (e.g., pitching in baseball) and lifelong physical activity (PA) movement skills (e.g., cycling and swimming) [7]. Fundamental movement skills are often described more precisely as basic stability (e.g., static balance), object control (also termed manipulative, e.g., throwing) or locomotor movements involving two or more body segments, (e.g., jumping) [5]. In this review, the global term "gross motor competence" will be used to reflect the various terminology used in the literature (e.g., fundamental movement/motor skills, stability skills, motor coordination) to define goal-directed human movement [8].
Emerging evidence supports associations between gross motor competence and a range of health outcomes. Children with low levels of gross motor competence tend to be less physically active and have lower levels of cardio-respiratory fitness [9]. A systematic review of 21 studies in children found strong evidence for positive associations between gross motor competency and time spent in PA, and cardio-respiratory fitness, and an inverse association with weight status [2]. More recent reviews have confirmed a positive association between gross motor competence and organized PA [10], and fitness [11]. Furthermore, there is longitudinal evidence that motor competence is important across the developmental lifespan [12]. For instance, higher gross motor competence attenuates the decline in PA levels throughout childhood [13], and motor competency in childhood is associated with higher levels of PA and fitness in adolescence $[14,15]$. In addition, longitudinal studies in children have demonstrated that lower motor competence is associated with increased body mass index (BMI) over time [16, 17].

Despite the health benefits associated with gross motor competence, motor competence in children and adolescents is low [9, 18, 19], with only $50 \%$ of children demonstrating competency in a broad range of skills [20-23]. While recent papers [24] and systematic reviews [25-28] indicate interventions can improve gross motor competence in both children and adolescents, published manuscripts lack important details (such as intervention intensity, duration, fidelity and characteristics of facilitators and participants) [27]. It remains unclear from these studies which correlates should be targeted to ensure interventions are optimized, and whether or not, and for whom, targeted and tailored interventions should be developed.

Understanding these important aspects of intervention development requires systematically reviewing the correlates of gross motor competence in children and adolescents. This will help to identify potential mechanisms of change by identifying the factors that are likely to make a difference and also target specific groups for intervention [29]. Ecological models are useful in framing potential influencing factors of health behavior (e.g., PA) [30] as they emphasize the environmental contexts of the behavior as well as the social and psychological influences. This can lead to an in-depth understanding of the multiple spheres of influence on behavior and can help guide intervention development.

Previous reviews of the pediatric correlates of motor competence have been limited by focusing only on fundamental movement skills [2] rather than using a broader definition of gross motor competence, examining only certain age groups (e.g., preschool) [31] and only documenting positive associations [31] (and not including null 
or negative associations). The current review will expand upon previous reviews $[2,31]$ in several important ways. Given that gross motor competence is important across the developmental lifespan [12], the age range from early childhood (age 3 years) to adolescence (up to age 18 years) will be reviewed. Furthermore, the review will identify gross motor competence as the outcome of interest and will endeavor to find which factors are reported as potential correlates of motor competence, using an ecological framework. Correlates will be categorized under five broader categories, namely (i) biological and demographic factors; (ii) behavioral attributes and skills; (iii) cognitive, emotional and psychological factors; (iv) cultural and social factors; and (v) physical environmental factors, as per a previous review on key correlates of PA [32], to understand the potential correlates of motor competency.

Whilst it is acknowledged that the association between gross motor competence and factors such as PA are likely to be reciprocal $[12,33]$, focusing on motor competence as the outcome (or dependent) variable will enable an examination of those factors that are potentially modifiable correlates of motor competence in young people. This will make the findings important and relevant to interventionists seeking to find ways of improving motor competence of children. This will also ensure this review does not replicate previous reviews that have examined the association between motor competence and PA or fitness [2, 10, 11]. Further, this review will also document null and negative correlates of gross motor competence, which will further help to isolate factors that are not important to target.

\section{Methods}

\subsection{Identification of Studies}

A structured electronic literature search was conducted in accordance with the Preferred Reporting Items for Systematic Reviews and Meta-Analyses (PRISMA) statement [34]. Six electronic databases (CINAHL Complete, ERIC, MEDLINE Complete, PsycINFO ${ }^{\circledR}$, Scopus and SPORTDiscus with Full Text) were searched from 1994 to 5 August 2014. Five of these databases (CINAHL Complete, ERIC, MEDLINE Complete, PsycINFO ${ }^{\circledR}$ and SPORTDiscus with Full Text) were accessed through the EBSCOhost platform.

The following search strings were used: ("motor skill*" OR "movement skill*" OR "motor development" OR "gross motor" OR "motor performance" OR "motor abilit*" OR “object manipulation" OR "motor coordination" OR "actual competence" OR "object control" OR "locomotor skill*" OR "motor proficiency" OR "motor competence") AND (preschool* OR kindergarten* OR child* OR adolescen* OR student* OR teen* OR youth) AND (correlate* OR determinant* OR predictor* OR relationship* OR association* ${ }^{*}$ OR difference*). An additional line of search terms was added to exclude studies with a focus on children and/or adolescents with a physical or cognitive impairment. These were as follows: AND NOT disabilit* OR disorder* OR impair* OR “cerebral palsy" OR autis*.

These strings were further limited to participants aged 3-18 years and English language. Only articles published in peer-reviewed journals were considered. Reviews, conference proceedings, and abstracts were not included. In addition to identifying studies through the database search, studies from authors' own bibliographic libraries were assessed for possible inclusion. After duplicates were removed, studies were initially assessed by screening titles and abstracts. If suitability could not be determined during this process, full-text articles were accessed and compared against inclusion criteria. The reference lists of retrieved full-text articles and other systematic reviews were also examined for relevant studies.

\subsection{Selection Criteria}

Two authors (SKL, SLCV) independently assessed the eligibility of studies for inclusion using the criteria below. Two other authors (LMB and ADO) were consulted when agreement could not be reached.

1. Participants were aged 3-18 years. The infant and toddler period were excluded so as to enable focus on motor competence rather than motor milestones or early developmental aspects. Studies with a focus on children and/or adolescents with a physical or cognitive impairment were excluded (e.g., cerebral palsy). Studies targeting overweight/obese children or children from schools in disadvantaged areas were included, but not those where study inclusion criteria specified that participants had developmental coordination delays.

2. Studies assessed gross motor competence. Motor competence was specified broadly as gross motor skill competency, encompassing fundamental movement skills and motor coordination.

3. Studies that used measurement batteries that were defined as "motor fitness" were excluded. Whilst physical fitness components such as cardiorespiratory endurance, body composition, muscular strength, endurance, and flexibility are sometimes termed "motor fitness" or "motor ability" [35], they were not considered as motor competence assessments for this review. Similarly, other performance-related components of fitness, such as agility [which can be defined 
as "a rapid whole-body movement with change of velocity or direction in response to a stimulus" [36] (p. 922)], were not considered as assessments of motor competence unless there was a clear distinction between the components being analyzed and discussed as aspects of fitness and those being analyzed and discussed as aspects of motor competence.

4. Studies that used measurement batteries that included fine motor skills as part of a composite score were excluded to preserve internal validity \{e.g., the Motoriktest für vier- bis sechsjährige Kinder 4-6 [37] and McCarron Assessment of Neuromuscular Development (MAND) [38]\}, unless analysis was conducted without the inclusion of fine motor skills. For some instruments, however, that assessed fine motor skills, one or more subtests may have met our inclusion criteria. For the Bruininks-Oseretsky Test of Motor Proficiency (BOTMP) [39], we only included the balance assessment, and only if more than two tests were used for this subtest. Other subtests in this assessment were excluded. Bilateral coordination (even in the short form of the assessment) contains both gross and fine motor elements. Manual coordination is a combination of two subtests: manual dexterity (which assesses fine motor skills) and upper limb coordination. The body coordination subtest includes bilateral coordination (which assesses fine motor skills). The agility component was excluded as this did not meet our criteria for a gross motor skill. For the Movement Assessment Battery for Children (M-ABC) [1], we included the balance subtest and the ball skills subtest. For the Peabody Developmental Motor Scales, Second Edition (PDMS-2) [40], we included the gross motor score and the subtests for object control, locomotor and stability.

5. Studies needed to assess a summary score of at least one aspect of gross motor competence. This could be an object control, locomotor, stability, or motor coordination summary score. At least two skill assessments needed to be included to make up a summary score. Studies that analyzed individual skills separately as the outcome variable were not included because the purpose of the review was to assess the factors that contributed to motor competence (or aspects of motor competence, defined above) more generally, rather than the factors that contributed to competency in one particular skill.

6. Studies presented a quantitative analysis of the association between a potential correlate and at least one aspect of gross motor competence as the outcome. Studies where gross motor competence was not treated as the outcome variable in analysis were excluded (i.e., correlation analysis). Studies that may have reported associations but still treated gross motor competence as the outcome or criterion variable were included.

7. Studies identified a potential correlate of gross motor competence that was not related to improvement as part of an intervention. For instance baseline associations would be potentially included, but not associations due to the impact of an intervention.

\subsection{Criteria for Risk of Bias Assessment}

Four authors (DPC, LLH, AZ, PJM) independently assessed the risk of bias in the studies that met the inclusion criteria. The criteria for assessing the risk of bias in the studies were adapted from the Strengthening the Reporting of Observation Studies in epidemiology (STROBE) statement [41] and previous reviews in similar areas [2, 27]. Four criteria were identified as being of most importance to this current review. These criteria were:

1. Were the participants likely to be representative of the population (i.e., country, state/region level)? Were schools or students randomly selected or were other data provided to indicate population representativeness?

2. Of those who consented to the study, did an adequate proportion have complete data for the outcome and all correlates of interest (i.e., no more than $20 \%$ of data was missing from a cross-sectional study and no more than $30 \%$ for a longitudinal study)?

3. (a) Did the study report the sources and details of motor competence assessment and were valid measures of motor competence used (validation in same age group published or validation data provided in the manuscript)?

4. (b) Did the study report adequate reliability of motor competence assessment [i.e., intra-class correlation (ICC) (or similar) $\geq 0.60]$ [42]? For studies that used process-oriented motor competence instruments (i.e., were concerned with the process or execution of the skill movement), adequate inter-rater reliability needed to be reported [i.e., ICC (or similar) $\geq 0.60$ ] in addition to the above validity and reliability measures.

5. Did the study report the sources and details of assessment of potential correlates and did all of the correlates of interest have acceptable validity and/or reliability? Acceptable validity was defined as $>0.40$, as per Brown et al. [42].

Initially five articles were sent to all authors to assess. After this assessment, any differences in risk of bias assessment were resolved via teleconference. Criteria were further refined, and two more studies were sent to all four authors. After a further discussion, the criteria were finalized and the remaining studies (including the five 
previously assessed) were divided among the four authors to score. Another author (SPS) then checked all studies to ensure consistency across the four raters in terms of criterion 3 only. This criterion was selected for an additional check because many studies used similar motor competence assessment instruments and we wanted to ensure raters had been comparable. Any differences at this point were resolved within the group of five authors facilitated by author LMB. Each criterion was scored as "yes" (a tick), "no" (a cross), "unclear" (?).

\subsection{Categorization of Variables and Level of Evidence}

Each correlate was summarized according to the state of evidence for that correlate. As per the review by Sallis and colleagues in 2000 [43], the percentages in parentheses refer to the number of associations supporting the expected association divided by the total number of associations for the variable. Based on the percentage of findings supporting the association, the variable was classified as no association (0-33\%), written as "0"; indeterminate/inconsistent (34-59\%), written as "?"; or a positive "+" or negative "-" association $(\geq 60 \%)$. When four or more studies found an association, it was classified as " ++ " or "- -" accordingly. When findings differed by sex, or age/ year, these were noted as "male" or "female" or the age/ year group. Summary codes were then based on the analysis that either supported the expected direction (either positive or negative) or did not (non-correlate). This meant that if a study found a positive association in one age group between a correlate and an aspect of motor competence and no association in another age group, the findings were counted in the summary codes as one study for and one study against. Some variables that were conceptually similar were combined for the purpose of summary codes if there were not enough studies to examine the variables individually (e.g., age and school year group).

\subsection{Meta-Analyses}

Meta-analyses were conducted to determine the relationship between gross motor competency and potential correlates using comprehensive meta-analysis software, version 2 for Windows (Biostat company, Englewood, NJ, USA) [44] with random effects models. Meta-analyses were conducted if at least three individual studies reported standardized coefficients. When studies compared reported multiple coefficients from the same study sample collected over different time points, the sample size was divided to avoid double counting. Heterogeneity was determined by Cochrane's $Q$ statistic and $I^{2}$ values (values of 25,50 and 75 were considered to indicate low, moderate and high heterogeneity, respectively) [45]. Publication bias was analyzed using Rosenthal's classic fail safe $N$ [46]. Correlations were interpreted as follows: $0-0.19$ (no correlation), 0.2-0.39 (low correlation), 0.4-0.59 (moderate correlation), $0.6-0.79$ (moderately high correlation) and $\geq 0.8$ (high correlation) [47].

\section{Results}

\subsection{Overview of Studies}

A total of 59 studies were identified (Fig. 1), published between 1995 and 2014. One author extracted descriptive data from the studies (HLB), and this was checked by two other authors (SKL and SLCV). Studies reflected the full range of ages, with one study in the birth to preschool age group [48] (included due to the upper age range), 19 [22, $49-66,93]$ in the preschool age group (defined as prior to school, 3-5 years), 29 [18, 67-92, 94, 95] in children (defined as primary, elementary school age), four in adolescents [33, 96-98], and five that covered a range of ages: preschool and children [23, 99]; children and adolescents $[19,100,101]$.

Most studies were conducted in the USA [18, 22, 51, 53, 58, 61-63, 66, 82, 87] and Australia [33, 49, 50, 70, 75, 76, $95,96,98,100]$. A total of 21 studies were conducted across Europe (Belgium [71-73, 90, 91], Finland [48, 88, 97, 99], Portugal [19, 64, 80], Denmark [59, 84], Greece [89, 93], Germany [101], Ireland [81], Italy [57], Northern Ireland [83] and Norway [92]), eight in Asia (Hong Kong [52, 55, 69], Bangladesh [85], India [67], Indonesia [68], Japan [54] and Pakistan [74]), five in Canada [56, 65, 77, $79,94]$, two in Brazil [23, 60] and a single study each from Israel [86] and South Africa [78]. Most study designs were cross-sectional $[18,19,22,23,33,49,50,52-71,74-80$, 83-90, 92-95, 98, 100, 101]. There were eight longitudinal studies $[48,72,73,81,82,91,96,97]$ and two randomized controlled trials (RCTs) [51, 99]. One RCT was included because they analyzed baseline data [51] and the other because they analyzed midline data only [99]. The sample sizes ranged from 34 [63] to $>5000$ [19]. More than half the studies had samples of $<300$ (Tables 1,2 ).

A range of instruments were used to assess gross motor competency. More than half (33 studies) used productoriented assessments (these are concerned with the outcome of movement, such as number of repetitions or whether the ball hits a target). The Körperkoordinationtest für Kinder (KTK) was used in eight studies [19, 72, 73, 80, $84,90,91,101]$. Several product-oriented assessments that use composite gross and fine motor batteries were included, with gross motor competence analyzed separately (as per our inclusion criteria): the BOTMP first edition, seven 
Studies identified through database searching

$(n=5,280)$

(CINAHL Complete $=281$, ERIC $=316$, MEDLINE Complete $=896$, PsycINFO=928, Scopus $=2,121$, SPORTDiscus with Full Text=738)
Additional studies identified through other sources (e.g. author's own bibliographic libraries) $(\mathrm{n}=13)$
Studies (titles \& abstracts) screened $(n=3,467)$

Full-text studies assessed for eligibility $(n=176)$

Studies after 1,826 duplicates removed $(n=3,467)$

Studies included in review $(\mathrm{n}=59)$

Fig. 1 Study progression during inclusion/exclusion

studies [55, 67, 74, 81, 86, 87, 93] and second edition, one study [85]; the M-ABC (six studies [48, 71, 77, 83, 92, 95]); and the PDMS-2 (three studies [51, 58, 64]). Five other studies used product-oriented tests particular to one study only [18, 54, 82, 94, 97], and three studies used a combination of different product assessments [59, 61, 99].

A total of 24 studies used process-oriented assessments. The most commonly used was the Test of Gross Motor 
Table 1 Descriptive information of included studies (ordered alphabetically)

\begin{tabular}{|c|c|c|c|c|c|c|c|}
\hline Study & Year & Country & Sample size & $\operatorname{Sex}(B, G)$ & $\begin{array}{l}\text { Age } \\
(\text { mean } \pm \mathrm{SD})\end{array}$ & Age group & Design \\
\hline Abbas et al. [67] & 2011 & India & 197 & B 99, G 98 & $9^{1 / 2}-14^{1 / 2} y$ & Children & Cross-sectional \\
\hline Bakhtiar [68] & 2014 & Indonesia & 67 & B 28, G 39 & $6.55 \pm 0.25 \mathrm{y}$ & Children & Cross-sectional \\
\hline Barnett et al. [96] & 2010 & Australia & 266 & $\begin{array}{l}\text { B } 48.1 \% \text {, } \\
\text { G } 51.9 \%\end{array}$ & $\begin{array}{l}\text { T1: } \\
10.06 \pm 0.63 y \\
\text { T2: } \\
16.44 \pm 0.64 y\end{array}$ & Adolescents & Longitudinal \\
\hline Barnett et al. [33] & 2011 & Australia & 215 & $\begin{array}{l}\text { B } 48.4 \% \text {, } \\
\text { G } 51.6 \%\end{array}$ & $16.4 \pm 0.6 \mathrm{y}$ & Adolescents & Cross-sectional \\
\hline Barnett et al. [50] & 2012 & Australia & 53 & B $41 \%$, G $59 \%$ & $4.15 \pm 0.72 \mathrm{y}$ & Preschool & Cross-sectional \\
\hline Barnett et al. [49] & 2013 & Australia & 76 & B $34, \mathrm{G} 42$ & $4.1 \pm 0.68 \mathrm{y}$ & Preschool & Cross-sectional \\
\hline $\begin{array}{l}\text { Bellows et al. } \\
\text { [51] }\end{array}$ & 2013 & USA & 263 & B NR, G NR & $\begin{array}{l}\text { C: } 51.5 \pm 6.6 \mathrm{mo} \\
\text { I: } 53 \pm 6.8 \mathrm{mo}\end{array}$ & Preschool & $\begin{array}{l}\text { Randomized } \\
\text { controlled trial } \\
\text { (baseline data } \\
\text { used) }\end{array}$ \\
\hline Choi Tse [69] & 2004 & Hong Kong & 90 & B 45, G 45 & $6-8 y$ & Children & Cross-sectional \\
\hline $\begin{array}{l}\text { Chow and Chan } \\
\text { [52] }\end{array}$ & 2011 & Hong Kong & 239 & B 121, G 118 & $3.6 \pm 0.2 \mathrm{y}$ & Preschool & Cross-sectional \\
\hline Cohen et al. [70] & 2014 & Australia & 460 & B $46 \%$, G $54 \%$ & $8.5 \pm 0.6 \mathrm{y}$ & Children & Cross-sectional \\
\hline $\begin{array}{l}\text { D'Hondt et al. } \\
\text { [71] }\end{array}$ & 2009 & Belgium & 117 & B 57, G 60 & $5-10 y$ & Children & Cross-sectional \\
\hline $\begin{array}{l}\text { D'Hondt et al. } \\
\text { [72] }\end{array}$ & 2013 & Belgium & 100 & B $52 \%$, G $48 \%$ & $\begin{array}{l}\mathrm{T} 1: 8.2 \pm 1.2 \mathrm{y} \\
\mathrm{T} 2: \mathrm{NR}\end{array}$ & Children & Longitudinal \\
\hline $\begin{array}{l}\text { D'Hondt et al. } \\
\text { [73] }\end{array}$ & 2014 & Belgium & $\begin{array}{l}\text { T1: } 2517 \\
\text { T2: } 754\end{array}$ & $\begin{aligned} & \text { T1: } \text { B } 52.8 \%, \\
& \text { G } 47.2 \% \\
& \text { T2: B } 50.8 \%, \\
& \text { G } 49.2 \%\end{aligned}$ & $\begin{array}{l}\text { T1: } 5-13 \text { y } \\
\text { T2: 7-13 y }\end{array}$ & Children & Longitudinal \\
\hline $\begin{array}{l}\text { Erwin and } \\
\text { Castelli [18] }\end{array}$ & 2008 & USA & 180 & B 87, G 93 & $10.45 \pm 0.78 y$ & Children & Cross-sectional \\
\hline $\begin{array}{l}\text { Goodway and } \\
\text { Rudisill [53] }\end{array}$ & 1997 & USA & 59 & B 30, G 29 & $4.74 \pm 0.31 \mathrm{y}$ & Preschool & Cross-sectional \\
\hline $\begin{array}{l}\text { Goodway et al. } \\
\text { [22] }\end{array}$ & 2010 & USA & $\begin{array}{c}469(\mathrm{MW}=275 \\
\mathrm{SW}=194)\end{array}$ & $\begin{array}{l}\text { MW: B 143, } \\
\text { G } 132 \\
\text { SW: B } 95, \text { G } 99\end{array}$ & $\begin{array}{l}\text { MW: } 54.80 \mathrm{mo} \\
\text { SW: } 56.37 \mathrm{mo}\end{array}$ & Preschool & Cross-sectional \\
\hline Habib et al. [74] & 1999 & Pakistan & 180 & B 90, G 90 & $5-13$ y & Children & Cross-sectional \\
\hline Hume et al. [75] & 2008 & Australia & 248 & B 123, G 125 & $\begin{array}{l}\text { B: } 10.1 \pm 0.44 \mathrm{y} \\
\text { G: } 10.0 \pm 0.28 \mathrm{y}\end{array}$ & Children & Cross-sectional \\
\hline Iteya et al. [54] & 1995 & Japan & 273 & $\begin{array}{l}\text { Foot laterality: } \\
\text { B 93, G } 42 \\
\text { Hand laterality: } \\
\text { B 78, G } 60\end{array}$ & $\begin{array}{l}\text { Foot laterality: } \\
5.3 \mathrm{y} \\
\text { Hand laterality: } \\
5.5 \mathrm{y}\end{array}$ & Preschool & Cross-sectional \\
\hline $\begin{array}{l}\text { Jaakkola and } \\
\text { Washington } \\
\text { [97] }\end{array}$ & 2013 & Finland & 152 & B 86, G 66 & $\begin{array}{l}\text { T1: } 13 \text { y } \\
\text { T2: NR } \\
\text { T3: NR }\end{array}$ & Adolescents & Longitudinal \\
\hline Jones et al. [76] & 2010 & Australia & 1299 & B $52 \%$, G $48 \%$ & $6.35 \pm 1.07 \mathrm{y}$ & Children & Cross-sectional \\
\hline $\begin{array}{l}\text { Junaid and } \\
\text { Fellowes [77] }\end{array}$ & 2006 & Canada & 103 & B 60, G 43 & $7-8 \mathrm{y}$ & Children & Cross-sectional \\
\hline $\begin{array}{l}\text { Kemp and } \\
\text { Pienaar [78] }\end{array}$ & 2013 & $\begin{array}{l}\text { South } \\
\text { Africa }\end{array}$ & 816 & B 419, G 397 & $6.84 \pm 0.39 \mathrm{y}$ & Children & Cross-sectional \\
\hline $\begin{array}{l}\text { Lam and Schiller } \\
\text { [55] }\end{array}$ & 2001 & Hong Kong & 320 & B 149, G 171 & $5-6 y$ & Preschool & Cross-sectional \\
\hline $\begin{array}{l}\text { Larouche et al. } \\
\text { [79] }\end{array}$ & 2014 & Canada & 491 & $\begin{array}{l}\text { B } 43.6 \% \text {, } \\
\text { G } 56.4 \%\end{array}$ & Grade $4-6$ & Children & Cross-sectional \\
\hline
\end{tabular}


Table 1 continued

\begin{tabular}{|c|c|c|c|c|c|c|c|}
\hline Study & Year & Country & Sample size & $\operatorname{Sex}(B, G)$ & $\begin{array}{l}\text { Age } \\
(\text { mean } \pm \mathrm{SD})\end{array}$ & Age group & Design \\
\hline $\begin{array}{l}\text { Laukkanen et al. } \\
\text { [99] }\end{array}$ & 2014 & Finland & 84 & B 38, G 46 & $\begin{array}{l}\text { Preschool B: } \\
5.92 \pm 0.45 \mathrm{y} \\
\text { Preschool G: } \\
5.95 \pm 0.47 \mathrm{y} \\
\text { Primary B: } \\
7.93 \pm 0.34 \mathrm{y} \\
\text { Primary G: } \\
8.06 \pm 0.15 \mathrm{y}\end{array}$ & $\begin{array}{l}\text { Preschool } \\
\text { and } \\
\text { children }\end{array}$ & $\begin{array}{l}\text { Randomized } \\
\text { controlled tria } \\
\text { (midline data } \\
\text { used) }\end{array}$ \\
\hline LeGear et al. [56] & 2012 & Canada & 260 & B $52 \%$, G $48 \%$ & 5 y 9 mo & Preschool & Cross-sectional \\
\hline Lopes et al. [80] & 2012 & Portugal & 213 & B 103, G 110 & $9.46 \pm 0.43 \mathrm{y}$ & Children & Cross-sectional \\
\hline Lopes et al. [19] & 2012 & Portugal & 7175 & B 3616, G 3559 & $6-14$ y & $\begin{array}{r}\text { Children and } \\
\text { adolescents }\end{array}$ & Cross-sectional \\
\hline $\begin{array}{l}\text { MacCobb et al. } \\
\text { [81] }\end{array}$ & 2005 & Ireland & 76 & B 38, G 38 & $8-10 y$ & Children & Longitudinal \\
\hline $\begin{array}{l}\text { McKenzie et al. } \\
{[82]}\end{array}$ & 2002 & USA & 207 & B 104, G 103 & $\begin{array}{l}\text { T1: } 4 \mathrm{y} \\
\text { T2: } 5 \mathrm{y} \\
\text { T3: } 6 \mathrm{y}\end{array}$ & Children & Longitudinal \\
\hline $\begin{array}{l}\text { McPhillips and } \\
\text { Jordan-Black } \\
\text { [83] }\end{array}$ & 2007 & $\begin{array}{r}\text { Northern } \\
\text { Ireland }\end{array}$ & 515 & B 283, G 232 & $\begin{array}{l}\text { Year } 1: \\
57.4 \pm 3.6 \mathrm{mo} \\
\text { Year } 4: \\
101.4 \pm 4.5 \mathrm{mo}\end{array}$ & Children & Cross-sectional \\
\hline $\begin{array}{l}\text { Morano et al. } \\
\text { [57] }\end{array}$ & 2011 & Italy & 80 & B 38, G 42 & $4.5 \pm 0.5 \mathrm{y}$ & Preschool & Cross-sectional \\
\hline $\begin{array}{l}\text { Morrison et al. } \\
\text { [84] }\end{array}$ & 2012 & Denmark & 498 & B 265, G 233 & $\begin{array}{l}\text { B: } 6.8 \pm 0.4 y \\
\text { G: } 6.7 \pm 0.4 y\end{array}$ & Children & Cross-sectional \\
\hline Nervik et al. [58] & 2011 & USA & 50 & B 26, G 24 & $53 \pm 10.5 \mathrm{mo}$ & Preschool & Cross-sectional \\
\hline Okely et al. [100] & 2004 & Australia & 4268 & B 2295, G 1973 & Grade $4,6,8,10$ & $\begin{array}{r}\text { Children and } \\
\text { adolescents }\end{array}$ & Cross-sectional \\
\hline Okely et al. [98] & 2001 & Australia & 2026 & B 1081, G 945 & $\begin{array}{l}\text { Year 8: } 13.3 \mathrm{y} \\
\text { Year 10: } 15.3 \mathrm{y}\end{array}$ & Adolescents & Cross-sectional \\
\hline Olesen et al. [59] & 2014 & Denmark & 607 & B 299, G 308 & $5.8 \pm 0.3 \mathrm{y}$ & Preschool & Cross-sectional \\
\hline Parvez et al. [85] & 2011 & Bangladesh & 303 & B $50 \%$, G $50 \%$ & $9.6 \pm 0.7 \mathrm{y}$ & Children & Cross-sectional \\
\hline $\begin{array}{l}\text { Queiroz et al. } \\
\text { [60] }\end{array}$ & 2014 & Brazil & $\begin{array}{l}\text { LOC SP: } 54 \\
\text { LOC NSP: } 54 \\
\text { OC SP: } 37 \\
\text { OC NSP: } 37\end{array}$ & $\begin{array}{l}\text { LOC SP: B } 30 \text {, } \\
\text { G } 24 \\
\text { LOC NSP: B } 30 \text {, } \\
\text { G } 24 \\
\text { OC SP: B } 17, \\
\text { G } 20 \\
\text { OC NSP: B } 17 \text {, } \\
\text { G } 20\end{array}$ & $\begin{array}{l}\text { LOC SP: } \\
60.0 \pm 8.7 \mathrm{mo} \\
\text { LOC NSP: } \\
59.4 \pm 8.1 \mathrm{mo} \\
\text { OC SP: } \\
60.9 \pm 7.9 \mathrm{mo} \\
\text { OC NSP: } \\
60.7 \pm 7.9 \mathrm{mo}\end{array}$ & Preschool & Cross-sectional \\
\hline Ratzon et al. [86] & 2000 & Israel & $\begin{array}{l}114 \text { (children born to } \\
\text { diabetic mothers }=57, \\
\text { children in control } \\
\text { group }=57 \text { ) }\end{array}$ & $\begin{array}{l}\text { Children born to } \\
\text { diabetic } \\
\text { mothers: } \\
\text { B } 51 \% \text {, } \\
\text { G } 49 \% \\
\text { Children in } \\
\text { control group: } \\
\text { B } 56 \% \text {, } \\
\text { G } 44 \%\end{array}$ & $\begin{array}{l}\text { Children born to } \\
\text { diabetic } \\
\text { mothers: } \\
8.09 \pm 1.77 \mathrm{y} \\
\text { Children in } \\
\text { control group: } \\
8.29 \pm 1.78 \mathrm{y}\end{array}$ & Children & Cross-sectional \\
\hline $\begin{array}{l}\text { Roberts et al. } \\
\text { [61] }\end{array}$ & 2012 & USA & 4650 & B 2150, G 2500 & 5 y $3 \mathrm{mo} \pm 4 \mathrm{mo}$ & Preschool & Cross-sectional \\
\hline Robinson [62] & 2010 & USA & 119 & B 65, G 54 & $4 \pm 0.55 y$ & Preschool & Cross-sectional \\
\hline
\end{tabular}


Table 1 continued

\begin{tabular}{|c|c|c|c|c|c|c|c|}
\hline Study & Year & Country & Sample size & $\operatorname{Sex}(B, G)$ & $\begin{array}{l}\text { Age } \\
(\text { mean } \pm \text { SD })\end{array}$ & Age group & Design \\
\hline $\begin{array}{l}\text { Robinson et al. } \\
\text { [63] }\end{array}$ & 2012 & USA & 34 & B 12, G 22 & $57 \pm 6.31 \mathrm{mo}$ & Preschool & Cross-sectional \\
\hline Roeber et al. [87] & 2012 & USA & $\begin{array}{l}67(\text { adopted }=33, \text { not } \\
\text { adopted }=34)\end{array}$ & $\begin{array}{l}\text { Adopted: B 16, } \\
\text { G } 17 \\
\text { Not adopted: } \\
\text { B 21, G } 13\end{array}$ & $\begin{array}{l}\text { Adopted: } 10 \mathrm{y} \\
9 \mathrm{mo} \pm 2 \mathrm{y} \\
2 \mathrm{mo} \\
\text { Not adopted: } 11 \mathrm{y} \\
2 \mathrm{mo} \pm 2 \mathrm{y} \\
1 \mathrm{mo}\end{array}$ & Children & Cross-sectional \\
\hline Saraiva et al. [64] & 2013 & Portugal & 367 & B 172, G 195 & $53 \pm 9.6 \mathrm{mo}$ & Preschool & Cross-sectional \\
\hline Slotte et al. [88] & 2014 & Finland & 304 & B 153, G 151 & $8.6 \pm 0.2 \mathrm{y}$ & Children & Cross-sectional \\
\hline $\begin{array}{l}\text { Spessato et al. } \\
\text { [23] }\end{array}$ & 2013 & Brazil & 1248 & B 641, G 607 & $3-10 y$ & $\begin{array}{l}\text { Preschool } \\
\text { and } \\
\text { children }\end{array}$ & Cross-sectional \\
\hline Temple et al. [65] & 2014 & Canada & 74 & B 41, G 33 & $\begin{array}{l}5 \mathrm{y} \\
11 \mathrm{mo} \pm 4 \mathrm{mo}\end{array}$ & Preschool & Cross-sectional \\
\hline $\begin{array}{l}\text { Tsapsakidou } \\
\text { et al. [89] }\end{array}$ & 2014 & Greece & 100 & B 54, G 46 & $8-9 y$ & Children & Cross-sectional \\
\hline $\begin{array}{l}\text { Vandendriessche } \\
\text { et al. [90] }\end{array}$ & 2012 & Belgium & 1955 & B $52 \%$, G $48 \%$ & $6-11$ y & Children & Cross-sectional \\
\hline $\begin{array}{l}\text { Vandorpe et al. } \\
\text { [91] }\end{array}$ & 2012 & Belgium & 371 & NR & $\begin{array}{l}\mathrm{T} 1: 8.3 \pm 1.1 \mathrm{y} \\
\mathrm{T} 2: 10.3 \pm 1.1 \mathrm{y}\end{array}$ & Children & Longitudinal \\
\hline $\begin{array}{l}\text { Vedul-Kjelsås } \\
\text { et al. [92] }\end{array}$ & 2013 & Norway & 67 & B 39, G 28 & $\begin{array}{l}\text { B: } \\
\quad 11.50 \pm 0.26 \mathrm{y} \\
\mathrm{G}: \\
11.40 \pm 0.26 \mathrm{y}\end{array}$ & Children & Cross-sectional \\
\hline $\begin{array}{l}\text { Venetsanou and } \\
\text { Kambas [93] }\end{array}$ & 2011 & Greece & 283 & B 145, G 138 & $61.77 \pm 5.43 \mathrm{mo}$ & Preschool & Cross-sectional \\
\hline $\begin{array}{l}\text { Viholanen et al. } \\
\text { [48] }\end{array}$ & 2006 & Finland & 130 & B 70, G 60 & $3.5 \mathrm{y}$ & $\begin{array}{l}\text { Birth to } \\
\text { preschool }\end{array}$ & Longitudinal \\
\hline Woll et al. [101] & 2013 & Germany & 4519 & B 2,310, G 2,209 & $4-17$ y & $\begin{array}{r}\text { Children and } \\
\text { adolescents }\end{array}$ & Cross-sectional \\
\hline $\begin{array}{l}\text { Woodard and } \\
\text { Yun [66] }\end{array}$ & 2001 & USA & 138 & B 65, G 73 & $5.3 \mathrm{y}$ & Preschool & Cross-sectional \\
\hline $\begin{array}{l}\text { Wright and Bos } \\
\text { [94] }\end{array}$ & 2012 & Canada & 84 & B 44, G 40 & $8-11$ y & Children & Cross-sectional \\
\hline Ziviani et al. [95] & 2009 & Australia & 124 & B 55, G 69 & $6-12 y$ & Children & Cross-sectional \\
\hline
\end{tabular}

$B$ boy, $C$ control, $G$ girl, $I$ intervention, $L O C$ locomotor skills, $m o$ months, $M W$ Midwestern, $N R$ not reported, $N S P$ no sports practice, $O C$ object control skills, $S P$ sports practice, $S W$ Southwestern, $T 1$ time point 1, T2 time point 2, T3 time point 3, $y$ years

Development (TGMD): first edition, three studies [53, 57, $66]$ and second edition, 15 studies [22, 23, 49, 50, 52, 56, $60,62,63,65,68-70,88,89]$. Australian resources were used in six studies (Department of Education Victoria [75, 98, 100] and Get Skilled Get Active [33, 76, 96]; three studies each). Two studies used both process and product assessment elements [78, 79].

Correlates were classified into the following five categories: (1) biological and demographic factors; (2) behavioral attributes and skills; (3) cognitive, emotional and psychological factors; (4) cultural and social factors; and
(5) physical environmental factors. In total, 49 correlates were assessed, with most studies assessing one (21 studies), two (21 studies) or three correlates (eight studies), and nine studies assessing between four and 12 correlates.

\subsection{Overview of Study Risk of Bias}

Study risk of bias is presented in Table 3 and shows that nearly one-third of studies (32\%) had samples that could be classed as representative of the study population, $58 \%$ of studies had minimal missing data, $86 \%$ used valid 
Table 2 Studies categorized by sample size

\begin{tabular}{|c|c|c|}
\hline $\begin{array}{l}\text { Total } \\
\text { sample }\end{array}$ & $\begin{array}{l}\text { No. of } \\
\text { studies }\end{array}$ & References \\
\hline$<100$ & 14 & $\begin{array}{l}{[49,50,53,57,58,63,65,68,69,81,87} \\
\quad 92,94,99]\end{array}$ \\
\hline 100-199 & 14 & $\begin{array}{l}{[18,48,60,62,66,67,71,72,74,77,86} \\
\quad 89,95,97]\end{array}$ \\
\hline $200-299$ & 10 & {$[33,51,52,54,56,75,80,82,93,96]$} \\
\hline $300-399$ & 5 & {$[55,64,85,88,91]$} \\
\hline $400-499$ & 4 & {$[22,70,79,84]$} \\
\hline $500-999$ & 3 & {$[59,78,83]$} \\
\hline $1000-2999$ & 4 & {$[23,76,90,98]$} \\
\hline $3000-5000$ & 4 & {$[61,73,100,101]$} \\
\hline$>5000$ & 1 & [19] \\
\hline
\end{tabular}

measures of gross motor competence, and $73 \%$ used reliable measures of motor competence. Most studies assessed potential correlates in a valid and reliable manner.

\subsection{Biological and Demographic Correlates}

The most commonly investigated biological and demographic correlates were sex (42 studies), age/grade (18 studies) and BMI (14 studies) (Table 4). There was strong evidence that being male was a positive correlate of object control competence and of motor coordination. The association between composite skill scores was indeterminate for boys, and there was no evidence that the sex of a child was associated with locomotor competence. There was inconsistent evidence for "being female" as associated with stability.

There was strong evidence for age (or grade) as a positive correlate of object control, locomotor skills and stability. There was inconsistent evidence for age as a correlate of motor coordination and skill composite.

There was strong evidence that higher BMI was negatively correlated with motor coordination and skill composite, whilst there was moderate evidence for stability. There was no evidence for BMI being negatively associated with object control skills and indeterminate evidence for locomotor skills. Similarly, other measures of adiposity, including higher waist circumference and percentage body fat, were negatively correlated with motor competence.

The socioeconomic background of the child was investigated in seven studies $[22,66,71,74,83,89,90]$ and showed inconsistent findings. A higher socioeconomic background was positively associated with locomotor, stability and skill composite, but one study showed a confounding effect with school year, with socioeconomic background positively influencing younger children but not older children's object control and stability [83]. Ethnicity was assessed in two studies, with both showing no association with motor composite score [18, 82]. Limb laterality (non-correlate) [54] and fitness (positive association) [18] were only assessed by one study each.

\subsection{Behavioral Attributes and Skills Correlates}

In terms of behavioral attributes and skills, different categories of PA and sport engagement constituted the majority of investigations in this category. Studies investigating PA were grouped together unless the particular type of PA was specified (e.g., dance, swimming). This resulted in studies addressing organized PA and sport, non-organized PA, and PA according to intensity being summarized together. There was inconsistent evidence for PA being a correlate of object control or locomotor skills. Only one study investigated PA as a correlate of stability competence [51]. There was, however, evidence for PA as a positive correlate of motor coordination [72, 90, 91] and skill composite [51, 84, 97]. Different types of classes (dance, "kindy gym," swimming) were investigated in one study, so a summary could not be calculated [49].

Sedentary time was only investigated in one study, with less time spent sedentary associated with better motor competence [80]. Early body and hand control were investigated in only one study, which found a positive result for body control and a negative result for hand control [48]. Likewise, the use of interactive and non-interactive electronic games was only investigated by one study, with a positive result for object control competence and no association for locomotor competence [50].

\subsection{Evidence for Other Factors as Correlates of Motor Competence}

Only one cognitive factor [18] and one psychological factor were investigated [33], with mixed results. One study examined the association between stability and a range of infant measures (study included because of the upper age range), including APGAR (Appearance, Pulse, Grimace, Activity and Respiration) and mental and motor development (Bayley Scales of Infant Development), with equivocal findings [81]. Similarly the studies that assessed a range of cultural and social factors (e.g., adoption status, parent skill confidence) $[49,86,87,89]$ and/or physical environmental factors (e.g., arsenic exposure, playground size at school) $[49,52,85]$ produced mixed results. No summary scores could be calculated for these factors because of the lack of studies assessing any one particular variable. 
Table 3 Risk of bias results

\begin{tabular}{|c|c|c|c|c|c|c|c|c|c|c|}
\hline \multicolumn{2}{|l|}{ Study details } & \multicolumn{4}{|l|}{ Study quality } & \multicolumn{5}{|c|}{ Correlates assessed and quality } \\
\hline Study & Year & $\begin{array}{l}\text { Representative } \\
\text { sampling }\end{array}$ & $\begin{array}{l}\text { Minimal } \\
\text { missing } \\
\text { data }\end{array}$ & $\begin{array}{l}\text { Valid } \\
\text { FMS }\end{array}$ & $\begin{array}{l}\text { FMS } \\
\text { reliabilities } \geq 0.60\end{array}$ & $\begin{array}{l}\text { Number } \\
\text { of } \\
\text { correlates }\end{array}$ & Age & Sex & BMI & Other correlates \\
\hline Abbas et al. [67] & 2011 & $\checkmark$ & $?$ & $\boldsymbol{V}$ & $\checkmark$ & 2 & $\checkmark$ & $\checkmark$ & & \\
\hline Bakhtiar [68] & 2014 & $\checkmark$ & $?$ & $\checkmark$ & $?$ & 1 & & $\checkmark$ & & \\
\hline Barnett et al. [96] & 2010 & $x$ & $x$ & $x$ & $\checkmark$ & 2 & $\checkmark$ & $\checkmark$ & & \\
\hline Barnett et al. [33] & 2011 & $\checkmark$ & $\checkmark$ & $x$ & $\checkmark$ & 2 & & & & $\begin{array}{l}\text { MVPA }(\boldsymbol{V}), \text { perceived } \\
\text { sports competence } \\
(\boldsymbol{V})\end{array}$ \\
\hline Barnett et al. [50] & 2012 & $x$ & $x$ & $\checkmark$ & $\checkmark$ & 5 & $\checkmark$ & $\boldsymbol{V}$ & & $\begin{array}{l}\text { PA }(\boldsymbol{}), \text { non- } \\
\text { interactive games } \\
(\boldsymbol{X}), \text { interactive } \\
\text { games }(\boldsymbol{x})\end{array}$ \\
\hline Barnett et al. [49] & 2013 & $x$ & $x$ & $\checkmark$ & $\checkmark$ & 12 & $\checkmark$ & $\checkmark$ & & $\begin{array}{l}\text { MVPA }(\boldsymbol{}), \\
\text { unstructured } \\
\text { activities }(\boldsymbol{X}), \\
\text { swimming lessons } \\
(\boldsymbol{X}) \text {, dance classes } \\
(\boldsymbol{X}) \text {, kindy gym } \\
\text { classes }(\boldsymbol{X}) \text {, parent } \\
\text { child interaction } \\
(\boldsymbol{X}) \text {, parent MPA/ } \\
\text { VPA }(\boldsymbol{X}) \text {, parent } \\
\text { skill confidence }(\boldsymbol{X}), \\
\text { visits to play spaces } \\
(\boldsymbol{X}), \text { toys home } \\
\text { equipment }(\boldsymbol{X})\end{array}$ \\
\hline $\begin{array}{l}\text { Bellows et al. } \\
\text { [51] }\end{array}$ & 2013 & $\checkmark$ & $\checkmark$ & $\checkmark$ & $\checkmark$ & 1 & & & & Steps $(\boldsymbol{V})$ \\
\hline Choi Tse [69] & 2004 & $x$ & $\checkmark$ & $\checkmark$ & $\checkmark$ & 1 & & $\checkmark$ & & \\
\hline $\begin{array}{l}\text { Chow and Chan } \\
\text { [52] }\end{array}$ & 2011 & $x$ & $\checkmark$ & $\checkmark$ & $\checkmark$ & 2 & & $\checkmark$ & & Preschool size $(\boldsymbol{})$ \\
\hline Cohen et al. [70] & 2014 & $\checkmark$ & $\checkmark$ & $\checkmark$ & $\checkmark$ & 1 & & $\checkmark$ & & \\
\hline $\begin{array}{l}\text { D'Hondt et al. } \\
\text { [71] }\end{array}$ & 2009 & $x$ & $\checkmark$ & $\checkmark$ & $\checkmark$ & 2 & & & $\checkmark$ & $\begin{array}{l}\text { SES/parental } \\
\text { education }(\boldsymbol{})\end{array}$ \\
\hline $\begin{array}{l}\text { D'Hondt et al. } \\
\text { [72] }\end{array}$ & 2013 & $\checkmark$ & $\checkmark$ & $\checkmark$ & $\checkmark$ & 2 & & & $\checkmark$ & Organized sport $(\boldsymbol{x})$ \\
\hline $\begin{array}{l}\text { D'Hondt et al. } \\
\text { [73] }\end{array}$ & 2014 & $\checkmark$ & $x$ & $\checkmark$ & $\checkmark$ & 3 & $\checkmark$ & & $\checkmark$ & PA (?) \\
\hline $\begin{array}{l}\text { Erwin and } \\
\text { Castelli [18] }\end{array}$ & 2008 & $x$ & $x$ & $\checkmark$ & $\checkmark$ & 6 & $\checkmark$ & $\checkmark$ & & $\begin{array}{l}\text { Ethnicity }(\boldsymbol{\sim}) \text {, school } \\
\text { year }(\boldsymbol{\sim}) \text {, fitness } \\
(\boldsymbol{\sim}) \text {, strategic } \\
\text { knowledge }(?)\end{array}$ \\
\hline $\begin{array}{l}\text { Goodway and } \\
\text { Rudisill [53] }\end{array}$ & 1997 & $x$ & $\checkmark$ & $\checkmark$ & $\checkmark$ & 1 & & $\checkmark$ & & \\
\hline $\begin{array}{l}\text { Goodway et al. } \\
\text { [22] }\end{array}$ & 2010 & $x$ & $\checkmark$ & $\checkmark$ & $\checkmark$ & 2 & & $\checkmark$ & & Region $(\boldsymbol{})$ \\
\hline Habib et al. [74] & 1999 & $x$ & $?$ & $\checkmark$ & $\checkmark$ & 3 & $\checkmark$ & $\checkmark$ & & $\operatorname{SES}(\boldsymbol{})$ \\
\hline Hume et al. [75] & 2008 & $x$ & $x$ & $\checkmark$ & $\checkmark$ & 2 & & $\checkmark$ & $\checkmark$ & \\
\hline Iteya et al. [54] & 1995 & $x$ & $\checkmark$ & $?$ & $?$ & 1 & & & & Limb laterality $(\boldsymbol{x})$ \\
\hline $\begin{array}{l}\text { Jaakkola and } \\
\text { Washington } \\
{[97]}\end{array}$ & 2013 & $x$ & $\checkmark$ & $\checkmark$ & $?$ & 3 & $\checkmark$ & $\checkmark$ & & PA (?) \\
\hline Jones et al. [76] & 2010 & $?$ & $\boldsymbol{\nu}$ & $x$ & ? & 1 & & & $\boldsymbol{\nu}$ & \\
\hline
\end{tabular}


Table 3 continued

\begin{tabular}{|c|c|c|c|c|c|c|c|c|c|c|}
\hline \multicolumn{2}{|l|}{ Study details } & \multicolumn{4}{|l|}{ Study quality } & \multicolumn{5}{|c|}{ Correlates assessed and quality } \\
\hline Study & Year & $\begin{array}{l}\text { Representative } \\
\text { sampling }\end{array}$ & $\begin{array}{l}\text { Minimal } \\
\text { missing } \\
\text { data }\end{array}$ & $\begin{array}{l}\text { Valid } \\
\text { FMS }\end{array}$ & $\begin{array}{l}\text { FMS } \\
\text { reliabilities } \geq 0.60\end{array}$ & $\begin{array}{l}\text { Number } \\
\text { of } \\
\text { correlates }\end{array}$ & Age & Sex & BMI & Other correlates \\
\hline $\begin{array}{l}\text { Junaid and } \\
\text { Fellowes [77] }\end{array}$ & 2006 & $x$ & $?$ & $\checkmark$ & $\checkmark$ & 1 & & $\checkmark$ & & \\
\hline $\begin{array}{l}\text { Kemp and } \\
\text { Pienaar [78] }\end{array}$ & 2013 & $\boldsymbol{V}$ & $\checkmark$ & $\checkmark$ & $?$ & 1 & & & $\checkmark$ & \\
\hline $\begin{array}{l}\text { Lam and Schiller } \\
\text { [55] }\end{array}$ & 2001 & $?$ & $\checkmark$ & $?$ & $?$ & 1 & & $\checkmark$ & & \\
\hline $\begin{array}{l}\text { Larouche et al. } \\
\text { [79] }\end{array}$ & 2014 & $x$ & $x$ & $\checkmark$ & $x$ & 1 & & $\checkmark$ & & \\
\hline $\begin{array}{l}\text { Laukkanen et al. } \\
\text { [99] }\end{array}$ & 2014 & $x$ & $\checkmark$ & $\checkmark$ & $\checkmark$ & 2 & $\checkmark$ & $\checkmark$ & & \\
\hline LeGear et al. [56] & 2012 & $x$ & $\boldsymbol{V}$ & $\checkmark$ & $\checkmark$ & 1 & & $\checkmark$ & & \\
\hline Lopes et al. [80] & 2012 & $x$ & $x$ & $\checkmark$ & $\checkmark$ & 2 & & $\checkmark$ & & $\begin{array}{l}\text { Sedentary behavior } \\
(\boldsymbol{N})\end{array}$ \\
\hline Lopes et al. [19] & 2012 & $x$ & $?$ & $\checkmark$ & $\checkmark$ & 2 & & $\checkmark$ & $\boldsymbol{V}$ & \\
\hline $\begin{array}{l}\text { MacCobb et al. } \\
\text { [81] }\end{array}$ & 2005 & $x$ & $x$ & $\checkmark$ & $\checkmark$ & 6 & & & & $\begin{array}{l}\text { Birth weight }(\boldsymbol{\sim}) \text {, } \\
\text { APGAR at } 5 \text { min } \\
(\boldsymbol{\sim}) \text {, Bailey infant } \\
\text { behavior }(\boldsymbol{}), \\
\text { Bailey mental }(\boldsymbol{\sim}) \text {, } \\
\text { NBAS motoric } \\
\text { cluster }(\boldsymbol{\sim}) \text {, Bailey } \\
\text { motor }(\boldsymbol{\sim})\end{array}$ \\
\hline $\begin{array}{l}\text { McKenzie et al. } \\
\text { [82] }\end{array}$ & 2002 & $x$ & $x$ & $x$ & $\checkmark$ & 2 & & $\checkmark$ & & Ethnicity $(\boldsymbol{})$ \\
\hline $\begin{array}{l}\text { McPhillips and } \\
\text { Jordan-Black } \\
\text { [83] }\end{array}$ & 2007 & $x$ & $\checkmark$ & $\checkmark$ & $\checkmark$ & 2 & & $\checkmark$ & & Disadvantage $(\boldsymbol{})$ \\
\hline $\begin{array}{l}\text { Morano et al. } \\
\text { [57] }\end{array}$ & 2011 & $x$ & $\checkmark$ & $\checkmark$ & $?$ & 2 & & $\checkmark$ & $\checkmark$ & \\
\hline $\begin{array}{l}\text { Morrison et al. } \\
\text { [84] }\end{array}$ & 2012 & $x$ & $x$ & $\checkmark$ & $?$ & 3 & & $\checkmark$ & & $\begin{array}{l}\text { PA }(\boldsymbol{\sim}) \text {, body fat } \\
\text { (skinfolds) (?) }\end{array}$ \\
\hline Nervik et al. [58] & 2011 & $x$ & $x$ & $x$ & $\checkmark$ & 3 & $\checkmark$ & $\checkmark$ & $\checkmark$ & \\
\hline Okely et al. [100] & 2004 & $\checkmark$ & $\checkmark$ & $\checkmark$ & $\checkmark$ & 2 & & & $\checkmark$ & Waist $(\boldsymbol{V})$ \\
\hline Okely et al. [98] & 2001 & $\checkmark$ & $\checkmark$ & $\checkmark$ & $\checkmark$ & 2 & $\checkmark$ & $\checkmark$ & & \\
\hline Olesen et al. [59] & 2014 & $\checkmark$ & $\checkmark$ & $\checkmark$ & $\checkmark$ & 1 & & $\checkmark$ & & \\
\hline Parvez et al. [85] & 2011 & $x$ & $\checkmark$ & $\checkmark$ & $x$ & 3 & & & & $\begin{array}{l}\text { Arsenic }(\boldsymbol{}), \\
\text { manganese }(\boldsymbol{}) \text {, } \\
\text { selenium }(\boldsymbol{})\end{array}$ \\
\hline $\begin{array}{l}\text { Queiroz et al. } \\
\text { [60] }\end{array}$ & 2014 & $\checkmark$ & $\checkmark$ & $\checkmark$ & $\checkmark$ & 2 & & $\checkmark$ & & Sport practice $(\boldsymbol{})$ \\
\hline Ratzon et al. [86] & 2000 & $x$ & $\checkmark$ & $\checkmark$ & $\checkmark$ & 1 & & & & Maternal diabetes $(\boldsymbol{})$ \\
\hline $\begin{array}{l}\text { Roberts et al. } \\
\text { [61] }\end{array}$ & 2012 & $\checkmark$ & $x$ & $\checkmark$ & $x$ & 1 & & & $\boldsymbol{V}$ & \\
\hline Robinson [62] & 2010 & $x$ & $\checkmark$ & $\checkmark$ & $\checkmark$ & 1 & & $\checkmark$ & & \\
\hline $\begin{array}{l}\text { Robinson et al. } \\
\text { [63] }\end{array}$ & 2012 & $x$ & $\checkmark$ & $\checkmark$ & $\checkmark$ & 1 & & $\checkmark$ & & \\
\hline
\end{tabular}


Table 3 continued

\begin{tabular}{|c|c|c|c|c|c|c|c|c|c|c|}
\hline \multicolumn{2}{|l|}{ Study details } & \multicolumn{4}{|l|}{ Study quality } & \multicolumn{5}{|c|}{ Correlates assessed and quality } \\
\hline Study & Year & $\begin{array}{l}\text { Representative } \\
\text { sampling }\end{array}$ & $\begin{array}{l}\text { Minimal } \\
\text { missing } \\
\text { data }\end{array}$ & $\begin{array}{l}\text { Valid } \\
\text { FMS }\end{array}$ & $\begin{array}{l}\text { FMS } \\
\text { reliabilities } \geq 0.60\end{array}$ & $\begin{array}{l}\text { Number } \\
\text { of } \\
\text { correlates }\end{array}$ & Age & Sex & BMI & Other correlates \\
\hline Roeber et al. [87] & 2012 & $x$ & $\checkmark$ & $\checkmark$ & $\checkmark$ & 4 & $\checkmark$ & & & $\begin{array}{l}\text { Adoption status }(\boldsymbol{}) \text {, } \\
\text { time living in USA } \\
(\boldsymbol{\sim}) \text {, Time spent } \\
\text { institutionalized } \\
\text { before adoption }(\boldsymbol{})\end{array}$ \\
\hline Saraiva et al. [64] & 2013 & $x$ & $?$ & $\checkmark$ & $\checkmark$ & 4 & $\checkmark$ & $\checkmark$ & $\checkmark$ & Height $(\boldsymbol{})$ \\
\hline Slotte et al. [88] & 2014 & $\checkmark$ & $x$ & $\checkmark$ & $?$ & 5 & & $\checkmark$ & $\checkmark$ & $\begin{array}{l}\text { Waist }(\boldsymbol{V}) \text {, height } \\
(\boldsymbol{\sim}) \text {, body fat }(\boldsymbol{})\end{array}$ \\
\hline $\begin{array}{l}\text { Spessato et al. } \\
\text { [23] }\end{array}$ & 2013 & $x$ & $?$ & $\checkmark$ & $\checkmark$ & 2 & $\checkmark$ & $\checkmark$ & & \\
\hline Temple et al. [65] & 2014 & $x$ & $\checkmark$ & $\checkmark$ & $?$ & 5 & $\checkmark$ & $\checkmark$ & & $\begin{array}{l}\text { Active physical } \\
\text { recreation (?), } \\
\text { participation in PA } \\
(?) \text {, organized sports } \\
(?)\end{array}$ \\
\hline $\begin{array}{l}\text { Tsapsakidou } \\
\text { et al. [89] }\end{array}$ & 2014 & $\checkmark$ & $\checkmark$ & $\checkmark$ & $\checkmark$ & 5 & & $\checkmark$ & & $\begin{array}{l}\text { Maternal education } \\
(\boldsymbol{V}), \text { paternal } \\
\text { education }(\boldsymbol{V}) \text {, SES } \\
(\boldsymbol{V}), \text { sport } \\
\text { participation }(\boldsymbol{V})\end{array}$ \\
\hline $\begin{array}{l}\text { Vandendriessche } \\
\text { et al. [90] }\end{array}$ & 2012 & $\checkmark$ & $\checkmark$ & $\checkmark$ & $\checkmark$ & 3 & $\checkmark$ & & & $\begin{array}{l}\text { SES }(\boldsymbol{}(), \text { sport } \\
\text { participation }(\boldsymbol{}(\boldsymbol{})\end{array}$ \\
\hline $\begin{array}{l}\text { Vandorpe et al. } \\
\text { [91] }\end{array}$ & 2012 & $\checkmark$ & $x$ & $\checkmark$ & $\checkmark$ & 1 & & & & Organized sport $(\boldsymbol{})$ \\
\hline $\begin{array}{l}\text { Vedul-Kjelsås } \\
\text { et al. [92] }\end{array}$ & 2013 & $x$ & $\checkmark$ & $\checkmark$ & $\checkmark$ & 1 & & $\checkmark$ & & \\
\hline $\begin{array}{l}\text { Venetsanou and } \\
\text { Kambas [93] }\end{array}$ & 2011 & $\checkmark$ & $\checkmark$ & $\checkmark$ & $\checkmark$ & 2 & $\checkmark$ & $\checkmark$ & & \\
\hline $\begin{array}{l}\text { Viholanen et al. } \\
\text { [48] }\end{array}$ & 2006 & $x$ & & $\checkmark$ & $\checkmark$ & 2 & & & & $\begin{array}{l}\text { Early body }(\boldsymbol{V}) \text {, hand } \\
\text { control }(\boldsymbol{V})\end{array}$ \\
\hline Woll et al. [101] & 2013 & $\checkmark$ & $?$ & $\checkmark$ & $x$ & 1 & & $\checkmark$ & & \\
\hline $\begin{array}{l}\text { Woodard and } \\
\text { Yun [66] }\end{array}$ & 2001 & $x$ & $\checkmark$ & $\checkmark$ & $x$ & 2 & & $\checkmark$ & & Group/SES $(\boldsymbol{})$ \\
\hline $\begin{array}{l}\text { Wright and Bos } \\
\text { [94] }\end{array}$ & 2012 & $x$ & $\checkmark$ & $?$ & $\checkmark$ & 3 & $\checkmark$ & $\checkmark$ & $\checkmark$ & \\
\hline Ziviani et al. [95] & 2009 & $x$ & $x$ & $\checkmark$ & $?$ & 1 & & $\checkmark$ & & \\
\hline Total & & $\begin{array}{l}32.2 \% \\
(19 / 59)\end{array}$ & $\begin{array}{l}57.6 \% \\
(34 / 59)\end{array}$ & $\begin{array}{c}86.4 \% \\
(51 / \\
59)\end{array}$ & $72.9 \%(43 / 59)$ & & 18 & 42 & 14 & \\
\hline
\end{tabular}

$\checkmark$ met criteria, $\boldsymbol{x}$ did not meet criteria, ? unclear whether it met criteria, APGAR Appearance, Pulse, Grimace, Activity and Respiration, BMI body mass index, FMS fundamental movement skills, MPA moderate physical activity, MVPA moderate to vigorous physical activity, NBAS Brazelton Neonatal Behavioral Assessment Scale, $P A$ physical activity, SES socioeconomic status, VPA vigorous physical activity

\subsection{Meta-Analysis of Motor Competence Correlates}

Two authors extracted data for the meta-analyses (NDR, ER). Meta-analyses were conducted for age and sex (Figs. 2, 3, 4, 5). No other correlates were investigated in three or more studies and reported standardized regression coefficients. The meta-analyses revealed small to medium effects for age and aspects of motor competence. For age, moderate effects were observed for object control skills $[r=0.37,95 \%$ confidence interval (CI) 0.29-0.35; Fig. 2, $Q=1.58, \quad I^{2}=0.000, \quad p=0.812, \quad$ classic fail safe $N=90]$, locomotor skills $(r=0.44,95 \%$ CI $0.37-0.51$; 


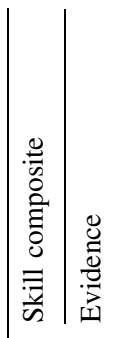
- .

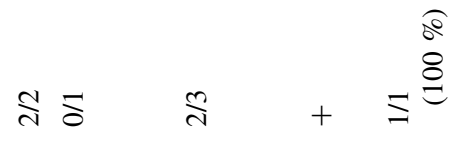

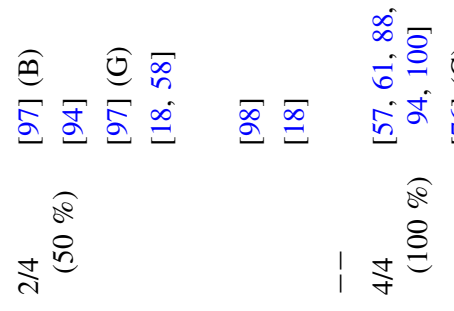

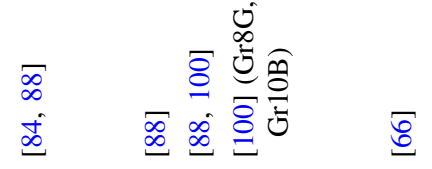

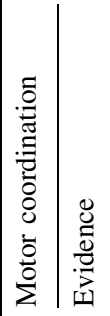

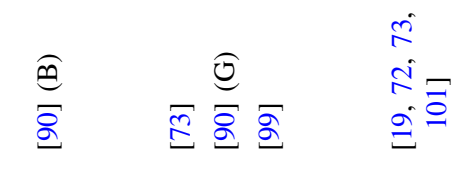
$\begin{array}{ll}\widehat{0} & \widehat{0} \\ \stackrel{\circ}{\alpha} & \stackrel{\alpha}{\alpha}\end{array}$
$+\frac{2}{a}$
$1 \frac{m^{2}}{\stackrel{5}{5}}$

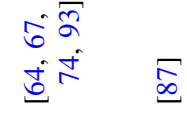

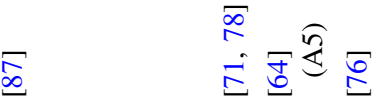

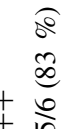
a. $\frac{a}{2}$
a. $\quad 5$ 告

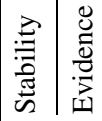
+ in
5
$\operatorname{mon}$
$\Xi 5$
$\stackrel{\text { I }}{\stackrel{5}{5}}$
वू.
iे
in
aे
वे

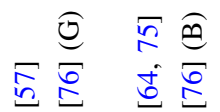
$\overline{\underline{\alpha}} \quad$ F
$+\frac{n}{5}$
$+\frac{5}{5}$
$\sqrt{6}$
요
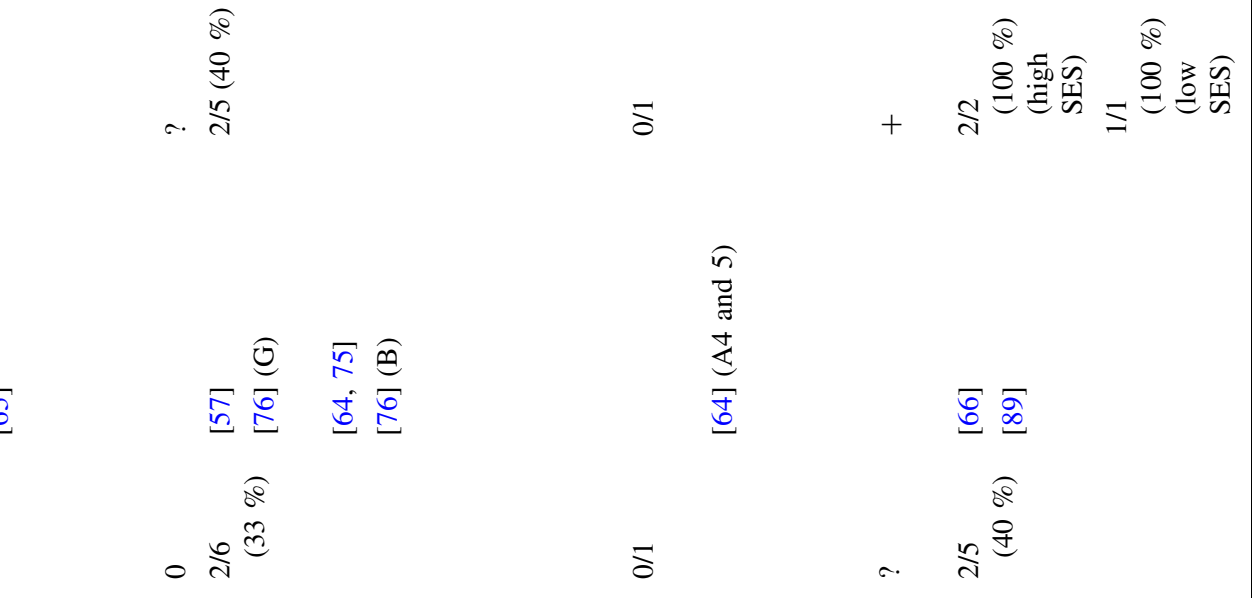

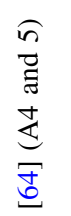

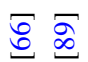
5
a. $\quad \frac{4}{\sqrt{2}}$

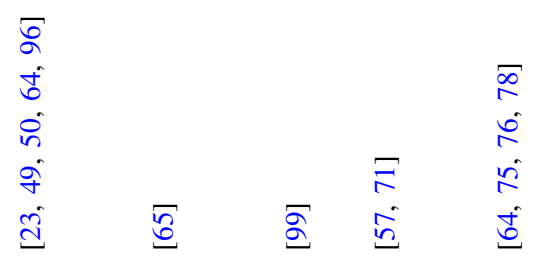

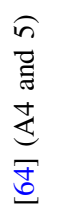

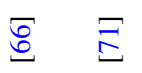

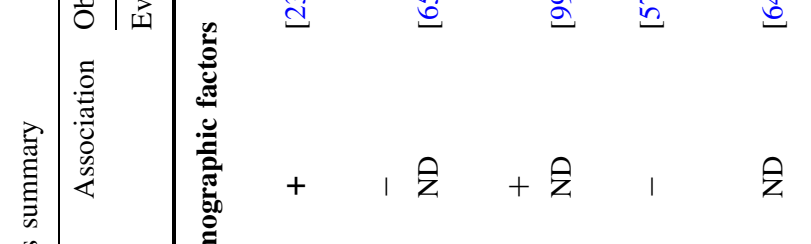
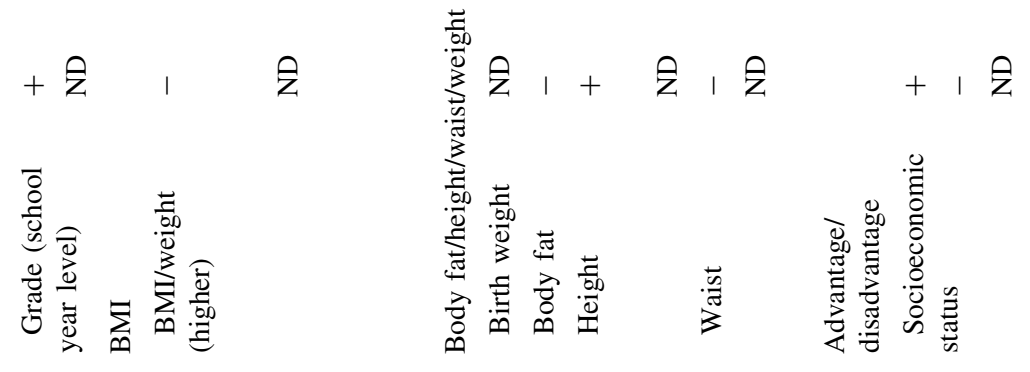

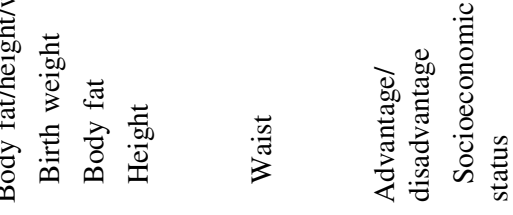




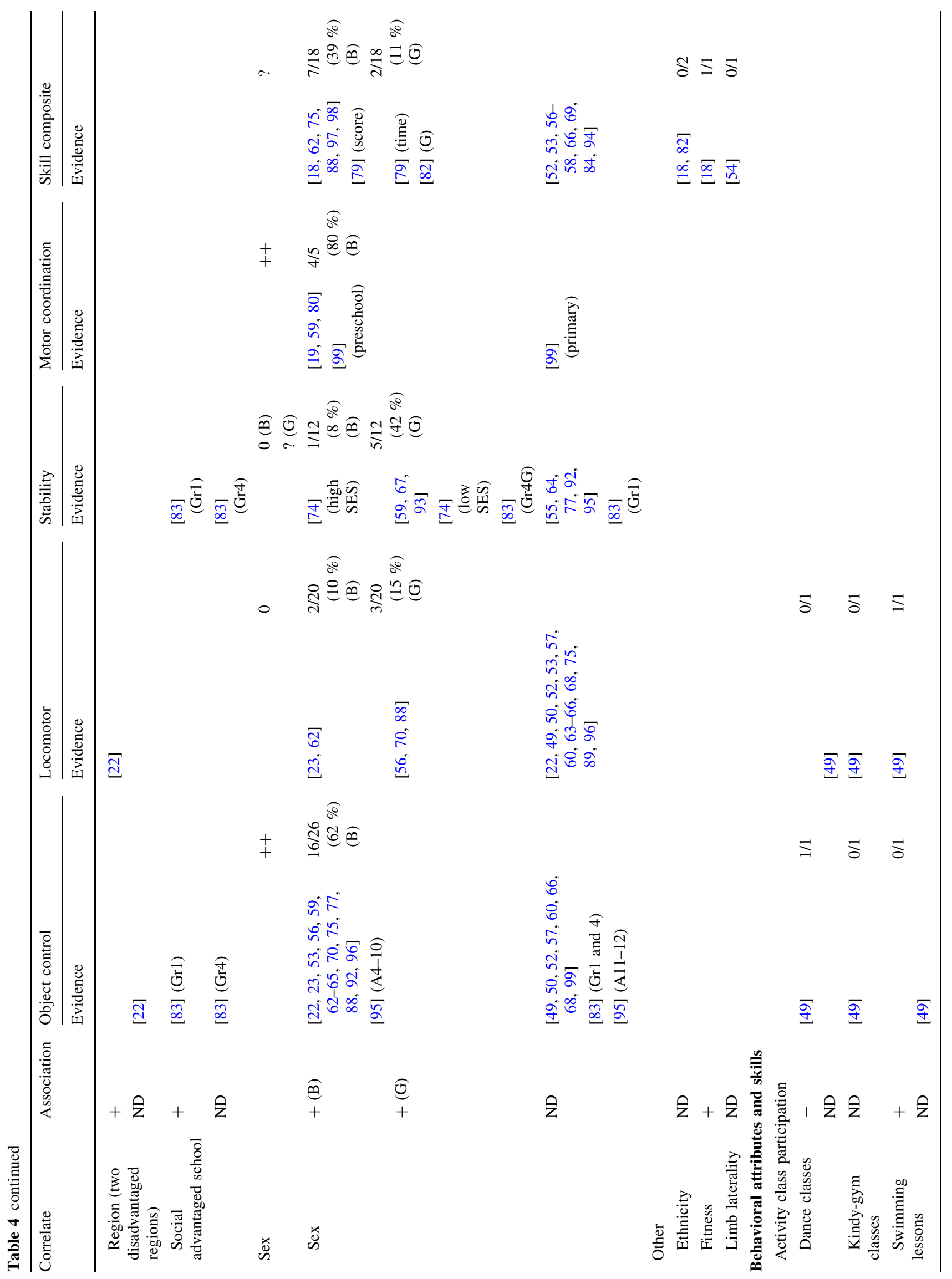




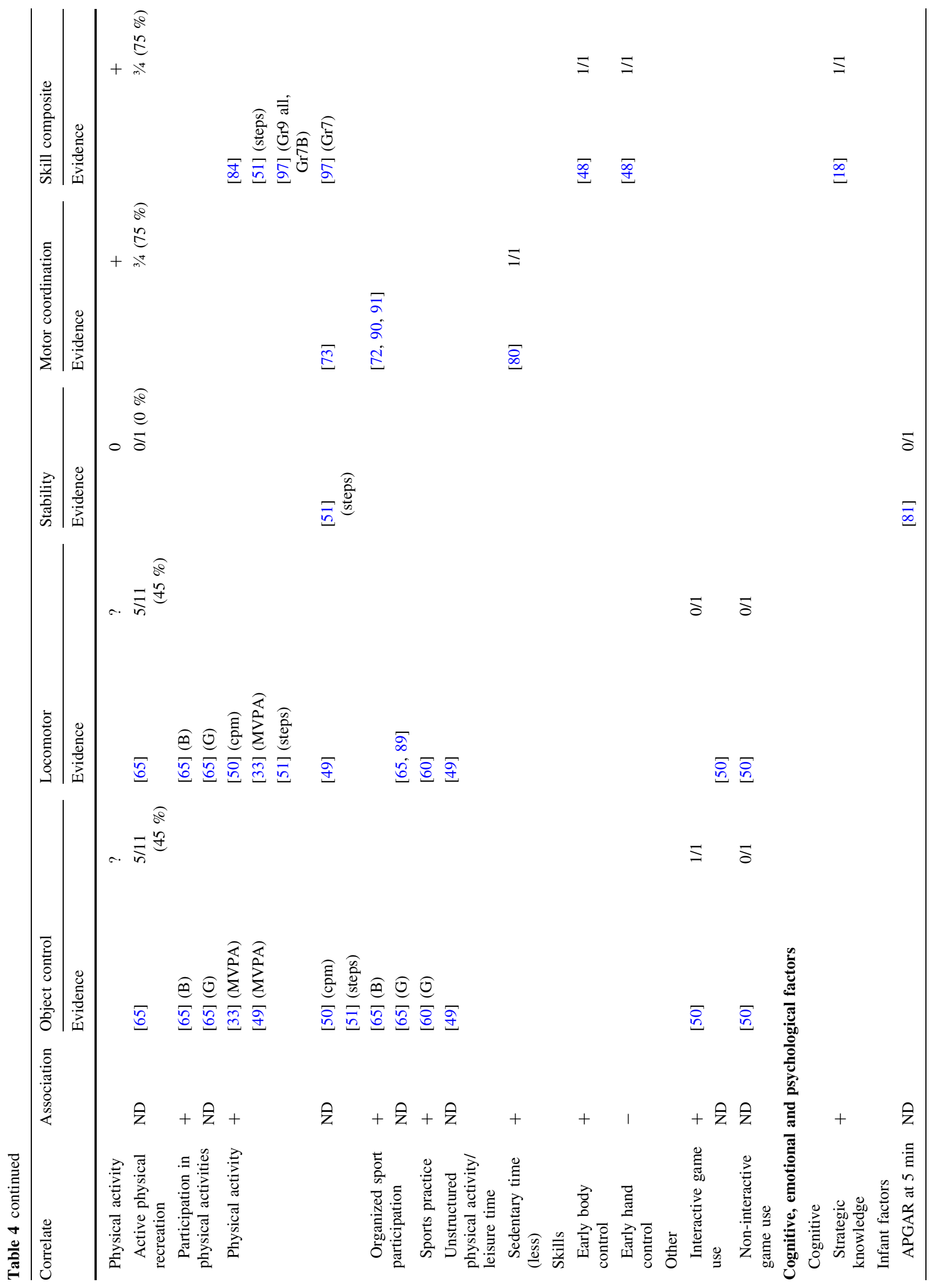




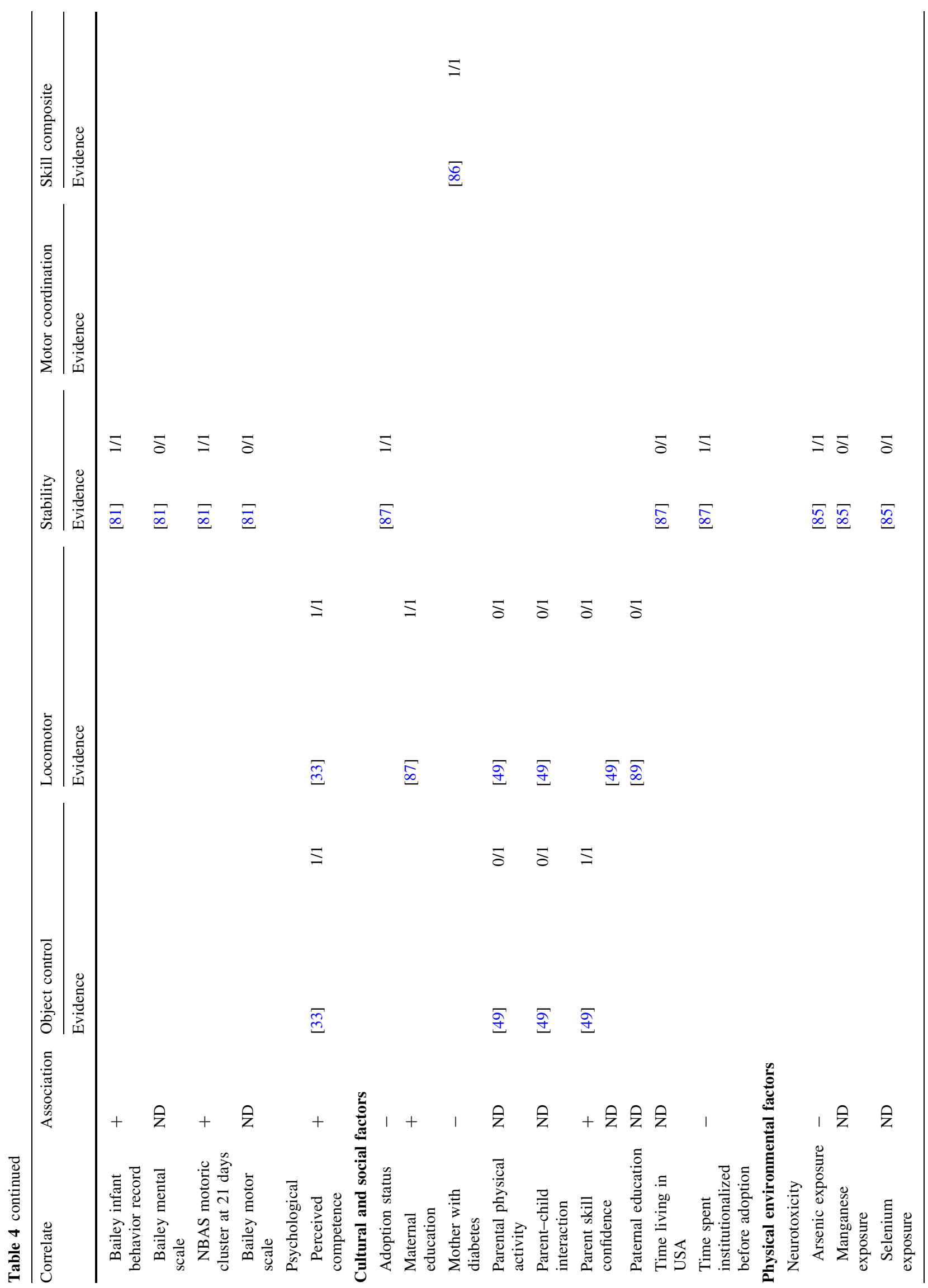




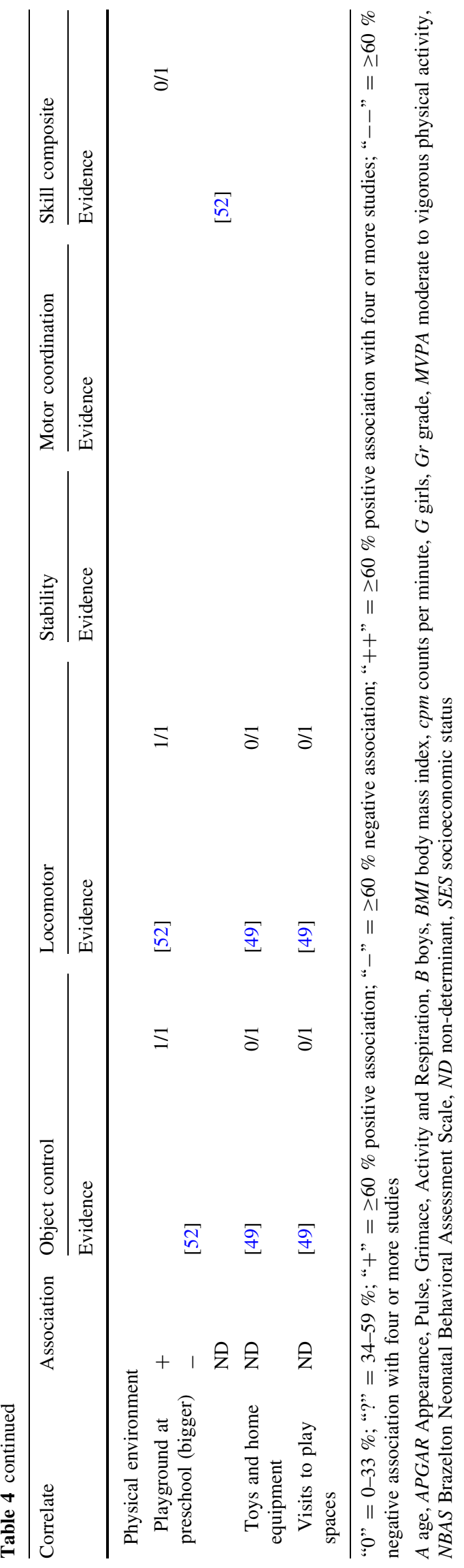

Fig. 3, $Q=3.913, I^{2}=23.38, p=0.271$, classic fail safe $N=104)$, and stability skills $(r=0.34,95 \% \quad \mathrm{CI}$ 0.29-0.39; Fig. 4, $Q=3.29, I^{2}=0.000, p=0.511$, classic fail safe $N=185$ ). A small effect was observed for the relationship between sex and object control skills, in favor of boys ( $r=0.23,95 \%$ CI 0.09-0.36; Fig. 5, $Q=13.515$, $I^{2}=70.40, p=0.009$, classic fail safe $N=40$ ). Low levels of heterogeneity were observed for the meta-analyses examining the relationship between skills and age. However, significant heterogeneity was found in the model that tested the relationship between sex and object control skills $\left(Q=13.515, I^{2}=70.402, p=0.009\right)$. For all metaanalyses, a large number of studies with an effect size of zero (classic fail safe $N$ values ranged from 40 to 185) would be required to cause the pooled point estimate to become statistically insignificant [46].

\section{Discussion}

\subsection{Overview of Findings}

It is clear from this review that investigating the correlates of gross motor competence is an emerging area, with the majority of studies $(69 \%)$ published in the last 5 years (since 2010). The most examined correlates of gross motor competency were biological and demographic factors, with age (positive), sex (boys more skilled than girls for object control and motor coordination), and adiposity (negative for motor coordination, stability, and skill composite) identified as correlates. In the behavioral attributes and skills category, PA and sport participation were the most investigated correlates, with some evidence for PA being a positive correlate of motor competence. Only one study examined cognitive, emotional and psychological factors as correlates of motor competence [18], precluding any conclusions regarding these outcomes. Similarly, only four studies [49, 86, 87, 89] investigated cultural and social factors that might contribute to motor competence, with mixed results. Finally, only three studies [49, 52, 85] investigated physical environment factors.

This review included only those studies for which gross motor competence was chosen as the outcome variable for the analysis, and therefore we did not include studies in which motor competence was a predictor or in which a simple bivariate analysis was conducted. With $83 \%$ (49/ 59) of the studies being cross-sectional, it could be argued that this is a matter of semantics, as either variable could be placed as the outcome. However, this assumption is not strictly correct. To illustrate, Barnett and colleagues [33], in a cross-sectional study, examined reciprocal associations between motor competence and PA in adolescents, using path analysis, and found a reciprocal relationship between 
Study

Saraiva et al [64] 3 years
Saraiva et al [64] 4 years
Saraiva et al [64] 5 years
Barnett et al [49]
Barnett et al [50]

Statistics for each study

$\begin{array}{rccrr}\text { Correlation } & \begin{array}{c}\text { Lower } \\ \text { limit }\end{array} & \begin{array}{c}\text { Upper } \\ \text { limit }\end{array} & \text { Z-Value } & \text { p-Value } \\ 0.428 & 0.272 & 0.562 & 5.011 & 0.000 \\ 0.371 & 0.207 & 0.515 & 4.250 & 0.000 \\ 0.301 & 0.130 & 0.454 & 3.388 & 0.001 \\ 0.350 & 0.135 & 0.533 & 3.122 & 0.002 \\ 0.430 & 0.181 & 0.627 & 3.252 & 0.001 \\ 0.372 & 0.292 & 0.446 & 8.567 & 0.000\end{array}$

Correlation and $95 \% \mathrm{Cl}$

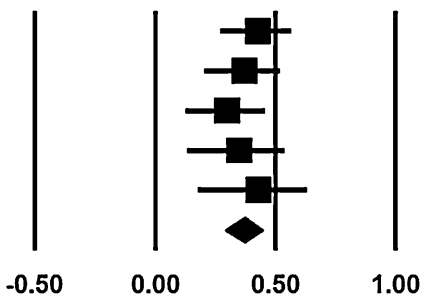

Fig. 2 Meta-analysis of the relationship between age and object control movement skill competency; final row indicates overall correlation coefficient, which can be interpreted as an effect size estimate. $Q=1.584, I^{2}=0.000, p=0.812$, classic fail safe $N=90$. CI confidence interval
Study

Saraiva et al [64] 3 years

Saraiva et al [64] 4 years

Barnett et al [49]

Barnett et al [50]
Statistics for each study

$\begin{array}{rccrr}\text { Correlation } & \begin{array}{c}\text { Lower } \\ \text { limit }\end{array} & \begin{array}{c}\text { Upper } \\ \text { limit }\end{array} & \text { Z-Value } & \text { p-Value } \\ 0.526 & 0.413 & 0.623 & 7.865 & 0.000 \\ 0.364 & 0.231 & 0.484 & 5.118 & 0.000 \\ 0.410 & 0.203 & 0.582 & 3.722 & 0.000 \\ 0.470 & 0.229 & 0.657 & 3.607 & 0.000 \\ 0.445 & 0.357 & 0.525 & 8.897 & 0.000\end{array}$

\section{Correlation and $95 \% \mathrm{Cl}$}
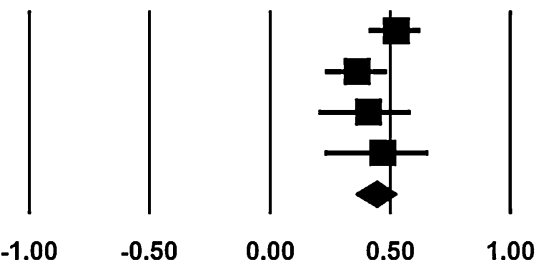

Fig. 3 Meta-analysis of the relationship between age and locomotor movement skill competency; final row indicates overall correlation coefficient, which can be interpreted as an effect size estimate.
$Q=3.913, I^{2}=23.382, p=0.271$, classic fail safe $N=104$. $C I$ confidence interval

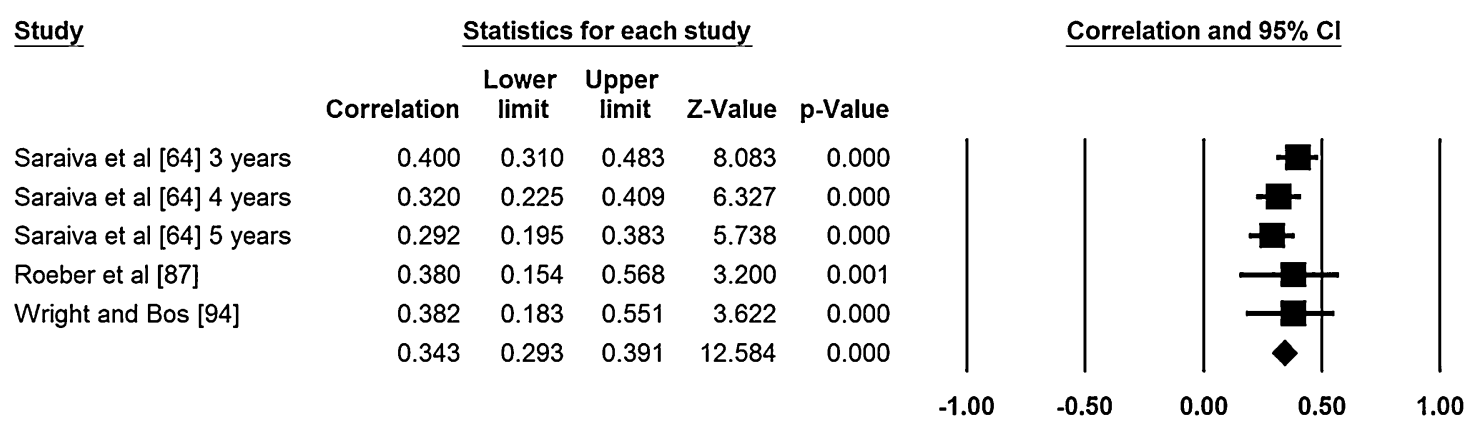

Fig. 4 Meta-analysis of the relationship between age and stability; final row indicates overall correlation coefficient, which can be interpreted as an effect size estimate. $Q=3.287, I^{2}=0.000, p=0.511$, classic fail safe $N=185$. $C I$ confidence interval

object control and moderate to vigorous physical activity (MVPA) and a one-way relationship from MVPA to locomotor skills. Restricting our included studies in this way is a study strength as we have isolated studies that hypothesized motor competence as the outcome in our effort to better understand what factors potentially influence motor competence. This does not mean that crosssectional evidence can be regarded as causal, but rather that the variables have been analyzed according to our hypothesis of interest.
Our meta-analyses of biological factors revealed smallto-medium effects for age and motor competence. The only other systematic review in this area (in preschool children only) also identified biological/demographic variables (such as sex and age) as having an association with motor competence [31]. Our meta-analysis showed that age was positively associated with locomotor, object control, and stability skills. It is not surprising that the older a child is, the better their skills, provided they continue to have opportunities to participate in activities that build compe- 
Study

Saraiva et al [64] 3 years

Saraiva et al [64] 4 years

Saraiva et al [64] 5 years

Barnett et al [49]

Barnett et al [50]
Statistics for each study

$\begin{array}{rrrrr}\text { Correlation } & \begin{array}{c}\text { Lower } \\ \text { limit }\end{array} & \begin{array}{c}\text { Upper } \\ \text { limit }\end{array} & \text { Z-Value } & \text { p-Value } \\ 0.227 & 0.052 & 0.388 & 2.531 & 0.011 \\ 0.353 & 0.187 & 0.499 & 4.024 & 0.000 \\ 0.470 & 0.319 & 0.598 & 5.564 & 0.000 \\ 0.030 & -0.242 & 0.298 & 0.212 & 0.832 \\ 0.080 & -0.148 & 0.300 & 0.685 & 0.493 \\ 0.282 & 0.198 & 0.362 & 6.368 & 0.000\end{array}$

Correlation and $95 \% \mathrm{Cl}$
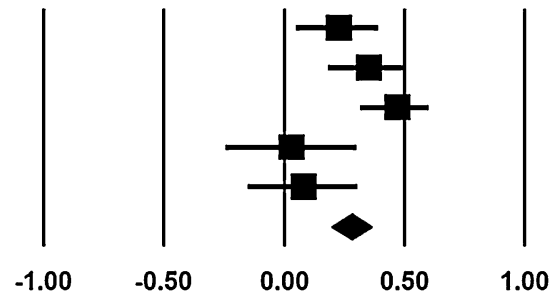

Fig. 5 Meta-analysis of the relationship between sex and object control movement skill competency; final row indicates overall correlation coefficient, which can be interpreted as an effect size

tence. Motor development in young children in the very early years is more influenced by biological maturation, and after this period, it becomes influenced more by practice and opportunity. Thus it is feasible that the relationship between age and gross motor competence might change across the developmental periods of early childhood, preschool, childhood and adolescence. Interestingly, even though the summary evidence confirmed age as a positive correlate of most aspects of motor competence, there were some studies (across all types of motor competence) that did not find this to be the case. For instance, when skill composite was the outcome in seven studies that investigated age, three reported a positive association, one study found a negative association for girls and three studies found no association. The study that found age to be a negative correlate was in the adolescent age group. This study suggested that girls' decline in their motor competence was due to reduced opportunity to be active, as the study also found that girls' PA declined during this period [97].

The null age findings in the other studies could be because many instruments (e.g., the TGMD) [102] provide the ability to age-standardize scores, so if these scores are being used in analyses, there may not be an age effect after standardizing. An alternate explanation for age not consistently being a correlate may be that some instruments used to assess motor competence have a ceiling effect and therefore fail to identify age differences in the older age groups. For example, instruments designed to assess motor competence via process-oriented assessments in young children have fewer and simpler assessment components than those designed for older children. Hence there may potentially be a certain age threshold where a motor competence assessment tool is no longer appropriate and a more complex assessment instrument is required. This underscores the need to ensure the instrument has been validated among the age group it purports to assess. This would suggest that the studies in older children and adolescents would be less likely to show positive associations estimate. $Q=13.515, I^{2}=70.402, p=0.009$, classic fail safe $N=40 . C I$ confidence interval

between age and gross motor competence. Yet of the studies that found age was not a correlate for at least one aspect of motor competence, two were in the early age groups (preschool), where skill trajectories are greater [58, 65], five were in primary/elementary school children [18, $73,87]$, and none were conducted in adolescents. It would therefore be unlikely that the children in these studies have all reached their maximum skill level or that the instruments used to assess motor competence had a ceiling effect. Furthermore, a different instrument to assess motor competence was used in each of these five studies, precluding the ability to find a pattern due to instrumentation. It is more likely that the age range investigated was not wide enough to show differences by age; this appears to be the case for four of the five studies [18, 58, 65, 87].

Weight status had differential associations with aspects of gross motor competence. Higher BMI was negatively correlated with motor coordination, stability, and skill composite, but not with object control skill competency. An indeterminate association was found for locomotor skills. An inverse relationship between body weight status and motor competence (defined broadly) has been found in other reviews, but these reviews did not examine associations for weight status with different categories of gross motor competence $[2,11]$. In contrast to object control skills, which tend to be more static, locomotor and stability skills involve shifting or controlling a larger body mass, which impedes functional movement [103] and contributes to the higher rate of lower limb problems among obese children (e.g., tibia varus, plantar pressure) [104]. The negative association between composite gross motor competence scores and higher BMI could reflect the composition of assessments where the composite comprises more motor coordination while moving and controlling the body compared with object control skills assessments.

Similarly, the sex of a child as a correlate of gross motor competence was also equivocal; sometimes males were 
favored, sometimes there were indeterminate results, and many studies showed no associations. This uncertainty is probably due to the fact that sex appears to relate differently to various aspects of gross motor competence. Being male was found to be a strong positive correlate of object control competence and motor coordination tasks. The meta-analyses confirmed this for object control skills, although the effect size was small. It is possible there is a biological basis for boys being more competent in object control skills. Butterfield et al. [105] allude to evolutionary/ biological differences pre-maturation for boys and girls, especially in reference to skills such as throwing and striking. Size and power might also be reflected in these findings, although considering only seven of the 59 studies included adolescents (and thus included males likely to have matured), this is unlikely to be the reason. The Iivonen and Sääkslahti [31] review also found being male to be a positive correlate for object control skills in the preschool age group, providing further support that size and strength due to maturation may not explain these findings. Productoriented assessments may be favorable towards size and power as they are concerned with the outcome of the movement (i.e., how far, how high), rather than the process of the movement. Although, of the ten studies that did not find sex to be a correlate of object control competence, seven used a process-oriented assessment [49, 50, 52, 57, $60,66,68]$ and three used a product-oriented assessment $[83,95,99]$, so there is no clear pattern favoring one type of assessment instrument over another. It is also likely that sociological factors may explain the difference in object control competence between girls and boys. Numerous studies have demonstrated that, compared with girls, boys receive greater encouragement, support and opportunities in PA and sports at home and in school and the broader community. As a result, girls' opportunities to enhance their gross motor competence may be limited, which would result in widening the gender gap [106-111]. Different PA and sport preferences between girls and boys may also help to explain this sex difference in object control skills. Being male is also a consistent positive correlate of PA in young children (aged 4-9 years) and a correlate for older children and adolescents [112].

We also found that sex was not a correlate of locomotor skills and had an indeterminate association for females for stability. In contrast, the Iivonen and Sääkslahti [31] review found that being female was a positive correlate for balance and locomotor skills. The three studies in our review that did find being female was a positive correlate for locomotor skills were all focused on children, but not as young as preschool children (ranged from 5-8 years) [56, $70,88]$; however, other studies with children of a similar age did not find a sex effect for these types of skills. A potential explanation for different findings between the reviews is that whilst the Iivonen and Sääkslahti [31] review found four studies that indicated locomotor skills were better performed by girls, this review only included studies that did find an association. Therefore these three studies may not represent the findings of the breadth of studies in the field.

We found that socioeconomic advantage was a positive correlate of locomotor skill stability and skill composite. Disadvantaged children may have less home sports equipment, reduced parental support and finances for organized sport and therefore be limited in terms of developing particular skills [113, 114]. There is a positive association between family support and PA in children and adolescents. Similarly, in adolescents, general social support for PA has been identified as a correlate [112].

Different aspects of PA and sport participation constituted the majority of investigations in the behavioral attributes and skills category. Interestingly, whilst we found PA to be a positive correlate of skill composite and motor coordination, we also found indeterminate evidence for PA being a correlate of object control or locomotor skill competence. Iivonen and Sääkslahti [31] found habitual PA to be a correlate of motor competence in preschool children, as have other reviews [2, 10] with PA (as the outcome). However these previous reviews did not attempt to see if different constructs of motor competence were predictive of PA. Our results suggest the relationship between PA and gross motor competence is not straightforward. It has been suggested that the relationship between motor competence and PA is not completely reciprocal [10]. In the model by Stodden et al. [12], it is postulated that in young children, PA is important to build motor competence but as children age, motor competence becomes more important for PA participation. Consequently, the relationship between PA and motor competence might vary depending on age of the child. It is logical that different types of PA may have differing associations with skills. It is also feasible that participation in the types of activities that use particular skills may lead to higher associations with that type of skill competence. For example, high participation in track events in athletics would likely be associated with better locomotor skills. Whilst we grouped PA factors together so as to provide a summary score, it is possible that if types of PA have differing associations with skill competence, these differences will be masked. One study was included that investigated different types of class participation in young children (dance, "kindy gym," swimming) and found the association was different according to object control and locomotor competence, which supports this hypothesis [49]. However, because only one study did this, summary scores could not be calculated. When examining PA intensity, rather than type of activity participation, there is also evidence (when PA 
intensity is the outcome) of differing associations. For example, MVPA and vigorous physical activity are commonly associated with motor competence [2, 33], whereas the few studies that investigate light activity did not find a relationship [115-117]. We would hypothesize a negative relationship between sedentary time and motor competence, and this was confirmed in the one study that investigated this [80]. Future research may seek to further investigate the nuances of the relationship between PA and gross motor competence to be able to tease out exactly what sorts of activity better contribute to what sorts of motor competence (and the reverse) at different ages. In order to understand the association between PA and motor competence, more appropriate and informative measures are required, particularly in children. Objective methods such as accelerometry, pedometers and global positioning systems are not yet sophisticated enough to comprehensively document the quality, context and type of activity. For example, some of the active play movements may register very little on an accelerometer (e.g., climbing, crawling, etc.). Direct observation would provide this information, but it is only a snapshot and costly.

There is a wide scope for future researchers to replicate studies and attempt to find evidence, particularly in the areas of cognitive, emotional and psychological factors. The link between cognition, PA and fitness has gained some attention recently, and this has extended to motor competence [118-120]. We only had one study that examined these aspects as correlates of motor competence [18], indicating this as an area of future research need. One study investigated infant factors (e.g., infant behavior record was a positive correlate) as predictors of motor competence in a longitudinal study [81]. This sort of investigation is quite unique in the literature but crucial to identifying the early life factors that contribute to better motor competence.

Only four studies investigated cultural and social factors that might contribute to motor competence, with these factors based on the parent [e.g., parent confidence (positive), mother with diabetes (negative)] or the child [e.g., adoption status (negative)]. One large study of correlates of gross motor competence in preschool children could not be included in this review as they used an assessment tool that includes fine motor skills in its composite score and thus did not meet our eligibility criteria [121]. Cools et al. [121] investigated the effects of a number of family and neighborhood characteristics on gross motor competence and identified factors such as father's PA and transport to school by bicycle as having a positive influence. This study [121] also identified some family factors negatively associated with preschool children's gross motor competence. Even though there is currently limited evidence in this area to draw any conclusions, the existing studies do point to the worthiness of future research in this area.
Only three studies in this review investigated physical environment factors (including such diverse factors as neurotoxicity, physical space, toys and equipment). Some positive and negative correlates were identified, again reinforcing the need for further research in this area.

\subsection{Strengths and Limitations}

This systematic review is the first to our knowledge that has investigated correlates of gross motor competence in children and adolescents. Using gross motor competence as a global definition is a study strength as a large number of studies could be included. A further strength is that we categorized correlates according to the specific ways motor competence has been defined and operationalized (object control, motor coordination, etc.), which enables us to have a greater understanding of which correlates influence specific types of motor competence. Indeed our findings do suggest that, once summary scores of motor competence are considered, evidence for some correlates differs according to how motor competence is operationalized. This shows that if we are seeking to provide a 'full' assessment of a child's motor competence, instruments should be used that can cover the broad spectrum of motor competence from motor coordination to fundamental movement skills. A recent validity investigation confirms this approach, finding that the KTK and the TGMD (version 2) measure discrete aspects of motor competence [122].

As stated in Sect. 4.1, it is a study strength that we isolated studies that hypothesized motor competence as the outcome in our effort to better understand what factors potentially influence motor competence. It is a further strength of this review that we conducted meta-analyses. However, very few studies focused on the same correlate and the same motor skill outcome, which meant we were limited by a lack of data for inclusion. Also, very few studies provided regression coefficients, and because of our exclusion criteria regarding correlation analysis, correlation data could not be used in our meta-analyses. Nevertheless we were able to provide support for some of the summary findings with regard to age and sex, which strengthens our results.

\section{Conclusions}

Age (increasing) is a correlate of children's gross motor competence. Weight status (healthy), sex (male) and socioeconomic background (higher) are consistent correlates for certain aspects of motor competence only. "Being male" as a correlate of object control skills and motor coordination has important intervention implications, as 
there is growing evidence of object control competence being a more salient predictor of PA and fitness behavior than locomotor competence [14, 70, 123]. Boys consistently have higher object control competence, which is a concern for females as their PA declines more than boys over adolescence, so if their object control competence is also lower, they may experience a negative spiral of engagement, ultimately resulting in an unhealthy weight status [12].

Somewhat in contrast to other reviews, we did not find PA to be a consistent positive predictor of motor competence $[2,10]$. The hypothesized Stodden model suggests that children who engage in more PA develop better motor competence and fitness and that this positive spiral of engagement ultimately impacts on weight status [12]. A narrative review has since examined the current state of evidence to support the hypothesized Stodden model [8]. Their conclusion was that the latest evidence indicates that motor competence is positively associated with multiple aspects of health (i.e., PA, cardiorespiratory fitness, muscular strength, muscular endurance and a healthy weight status). Based on the evidence in this current review, we can confirm that both PA and weight status are important to motor competence, but this relationship does appear to depend on the way motor competence is operationalized. This finding also has important intervention implications, suggesting that addressing childhood and adolescent obesity prevention through motor competence interventions, such as those tested in after-school settings [124], requires further investigation.

The authors of the aforementioned narrative review also concluded that there are still questions remaining related to the increased strength of associations across time and in terms of the direction of associations (i.e., what is the antecedent and what is the consequent [8]). This current systematic review has contributed to this understanding by specifically highlighting the factors that predict gross motor competence. Future researchers may seek to investigate the role of many correlates of motor competence that could not be evaluated due to the small number of studies for each correlate, so as to build the knowledge base in this area.

\section{Compliance with Ethical Standards}

Funding The preparation of this review was funded by the Australasian Child and Adolescent Obesity Research Network (ACAORN). Lisa Barnett was supported by an Alfred Deakin postdoctoral fellowship. Samuel Lai and Sanne Veldman were supported by ACAORN funds for review tasks associated with the preparation of this manuscript. Dylan Cliff is funded by an Australian Research Council Discovery Early Career Researcher Award (DE140101588). Nicola Ridgers is funded by an Australian Research Council Discovery Early Career Researcher Award (DE120101173). Anthony Okely is supported by a Career Development Fellowship from the National Heart Foundation of Australia.
Conflict of interest Lisa Barnett, Samuel Lai, Sanne Veldman, Louise Hardy, Dylan Cliff, Philip Morgan, Avigdor Zask, Sarah Shultz, David Lubans, Nicola Ridgers, Elaine Rush, Helen Brown and Anthony Okely declare that they have no conflicts of interest relevant to the content of this review.

Open Access This article is distributed under the terms of the Creative Commons Attribution 4.0 International License (http:// creativecommons.org/licenses/by/4.0/), which permits unrestricted use, distribution, and reproduction in any medium, provided you give appropriate credit to the original author(s) and the source, provide a link to the Creative Commons license, and indicate if changes were made.

\section{References}

1. Henderson S, Sugden D. Movement assessment battery for children. London: The Psychological Corporation; 1992.

2. Lubans DR, Morgan PJ, Cliff DP, et al. Fundamental movement skills in children and adolescents: review of associated health benefits. Sports Med. 2010;40(12):1019-35.

3. Branta C, Haubenstricker J, Seefeldt V. Age changes in motor skills during childhood and adolescence. Exerc Sport Sci Rev. 1984;12(1):467-520.

4. Gallahue D, Ozmun J. Understanding motor development: infants, children, adolescents, adults. 6th ed. Boston: McGrawHill; 2006.

5. Gallahue DL, Cleland-Donnelly F. Developmental physical education for all children. 4th ed. Champaign: Human Kinetics; 2003.

6. Clarke JE, Metcalfe JS. The mountain of motor development: a metaphor. In: Clarke JE, Humphrey JH, editors. Motor development: research and reviews, vol. 2. Reston: National Association for Sport and Physical Education; 2002. p. 163-90.

7. Hulteen RM, Lander NJ, Morgan PJ, et al. Validity and reliability of field-based measures for assessing movement skill competency in lifelong physical activities: a systematic review. Sports Med. 2015;45(10):1443-54.

8. Robinson LE, Stodden DF, Barnett LM, et al. Motor competence and its effect on positive developmental trajectories of health. Sports Med. 2015;45(9);1273-84.

9. Hardy LL, Reinten-Reynolds T, Espinel P, et al. Prevalence and correlates of low fundamental movement skill competency in children. Pediatrics. 2012;130(2):e390-8.

10. Holfelder B, Schott N. Relationship of fundamental movement skills and physical activity in children and adolescents: a systematic review. Psychol Sport Exerc. 2014;15(4):382-91.

11. Cattuzzo MT, dos Santos Henrique R, Ré AHN, et al. Motor competence and health related physical fitness in youth: a systematic review. J Sci Med Sport. 2016;19(2):123-9.

12. Stodden DF, Goodway JD, Langendorfer SJ, et al. A developmental perspective on the role of motor skill competence in physical activity: an emergent relationship. Quest. 2008;60(2): 290-306.

13. Lopes VP, Rodrigues LP, Maia JAR, et al. Motor coordination as predictor of physical activity in childhood. Scand J Med Sci Sports. 2011;21(5):663-9.

14. Barnett LM, van Beurden E, Morgan PJ, et al. Childhood motor skill proficiency as a predictor of adolescent physical activity. J Adolesc Health. 2009;44(3):252-9.

15. Barnett LM, van Beurden E, Morgan PJ, et al. Does childhood motor skill proficiency predict adolescent fitness? Med Sci Sports Exerc. 2008;40(12):2137-44. 
16. Lopes VP, Maia JAR, Rodrigues LP, et al. Motor coordination, physical activity and fitness as predictors of longitudinal change in adiposity during childhood. Eur J Sport Sci. 2012;12(4):384-91.

17. Martins D, Maia J, Seabra A, et al. Correlates of changes in BMI of children from the Azores islands. Int J Obes. 2010;34(10): 1487-93.

18. Erwin HE, Castelli DM. National physical education standards: a summary of student performance and its correlates. Res Q Exerc Sport. 2008;79(4):495-505.

19. Lopes VP, Stodden DF, Bianchi MM, et al. Correlation between BMI and motor coordination in children. J Sci Med Sport. 2012;15(1):38-43.

20. Bryant ES, Duncan MJ, Birch SL. Fundamental movement skills and weight status in British primary school children. Eur J Sport Sci. 2013;14(7):730-6.

21. Hardy LL, Barnett L, Espinel P, et al. Thirteen-year trends in child and adolescent fundamental movement skills: 1997-2010. Med Sci Sports Exerc. 2013;45(10):1965-70.

22. Goodway JD, Robinson LE, Crowe H. Gender differences in fundamental motor skill development in disadvantaged preschoolers from two geographical regions. Res Q Exerc Sport. 2010;81(1):17-24.

23. Spessato BC, Gabbard C, Valentini N, et al. Gender differences in Brazilian children's fundamental movement skill performance. Early Child Dev Care. 2013;183(7):916-23.

24. Cohen KE, Morgan PJ, Plotnikoff RC, et al. Physical activity and skills intervention: SCORES cluster randomized controlled trial. Med Sci Sports Exerc. 2015;47(4):765-74.

25. Lai SK, Costigan SA, Morgan PJ, et al. Do school-based interventions focusing on physical activity, fitness, or fundamental movement skill competency produce a sustained impact in these outcomes in children and adolescents? A systematic review of follow-up studies. Sports Med. 2014;44(1):67-79.

26. Logan SW, Robinson LE, Wilson AE, et al. Getting the fundamentals of movement: a meta-analysis of the effectiveness of motor skill interventions in children. Child Care Health Dev. 2012;38(3):305-15.

27. Morgan PJ, Barnett LM, Cliff DP, et al. Fundamental movement skill interventions in youth: a systematic review and metaanalysis. Pediatrics. 2013;132(5):e1361-83.

28. Riethmuller AM, Jones RA, Okely AD. Efficacy of interventions to improve motor development in young children: a systematic review. Pediatrics. 2009;124(4):E782-92.

29. Sallis JF, Owen N, Fisher E. Ecological models of health behavior. In: Glanz K, Rimer B, Viswanath K, editors. Health behavior and health education: theory, research, and practice. San Francisco: Jossey-Bass; 2008. p. 465-86.

30. Welk GJ. The youth physical activity promotion model: a conceptual bridge between theory and practice. Quest. 1999;51(1): $5-23$.

31. Iivonen S, Sääkslahti AK. Preschool children's fundamental motor skills: a review of significant determinants. Early Child Dev Care. 2013;184(7):1107-26.

32. Sterdt E, Liersch S, Walter U. Correlates of physical activity of children and adolescents: a systematic review of reviews. Health Educ J. 2014;73(1):72-89.

33. Barnett LM, Morgan PJ, Van Beurden E, et al. A reverse pathway? Actual and perceived skill proficiency and physical activity. Med Sci Sports Exerc. 2011;43(5):898-904.

34. Moher D, Liberati A, Tetzlaff J, et al. Preferred reporting items for systematic reviews and meta-analyses: the PRISMA statement. J Clin Epidemiol. 2009;62(10):1006-12.

35. Pate RR. The evolving definition of physical fitness. Quest. 1988;40(3):174-9.

36. Sheppard JM, Young WB. Agility literature review: classifications, training and testing. J Sports Sci. 2006;24(9):919-32.
37. Zimmer R, Volkamer M. Motoriktest fur vier- bissechsjarige Kinder (manual). Weinheim: Betltztest; 1987.

38. McCarron L. McCarron assessment of neuromuscular development. 3rd ed. Dallas: McCarron-Dial Systems Inc; 1997.

39. Bruininks R. Bruininks-Oseretsky test of motor proficiency. Circle Pines: American Guidance Service; 1978.

40. Folio A, Fewell R. Peabody developmental motor scales. 2nd ed. Austin: Pro-Ed; 2000.

41. von Elm E, Altman DG, Egger M, et al. The Strengthening the Reporting of Observational Studies in Epidemiology (STROBE) statement: guidelines for reporting observational studies. Prev Med. 2007;45(4):247-51.

42. Brown H, Hume C, ChinApaw M. Validity and reliability of instruments to assess potential mediators of children's physical activity: a systematic review. J Sci Med Sport. 2009;12(5): 539-48.

43. Sallis JF, Prochaska JJ, Taylor WC. A review of correlates of physical activity of children and adolescents. Med Sci Sports Exerc. 2000;32(5):963-75.

44. Borenstien M, Hedges L, Higgins J, et al. Comprehensive meta analysis version 2. Englewood: Biostat; 2005.

45. Higgins JPT, Thompson SG, Deeks JJ, et al. Measuring inconsistency in meta-analyses. BMJ. 2003;327(7414):557-60.

46. Rosenthal $\mathrm{R}$. The file drawer problem and tolerance for null results. Psychol Bull. 1979;86(3):638-41.

47. Cohen J. A power primer. Psychol Bull. 1992;112(1):155-9.

48. Viholanen $\mathrm{H}$, Ahonen $\mathrm{T}$, Cantell $\mathrm{M}$, et al. The early motor milestones in infancy and later motor skills in toddlers: a structural equation model of motor development. Phys Occup Ther Pediatr. 2006;26(1-2):91-113.

49. Barnett L, Hinkley T, Okely AD, et al. Child, family and environmental correlates of children's motor skill proficiency. J Sci Med Sport. 2013;16(4):332-6.

50. Barnett LM, Hinkley T, Okely AD, et al. Use of electronic games by young children and fundamental movement skills? Percept Mot Skills. 2012;114(3):1023-34.

51. Bellows LL, Davies PL, Anderson J, et al. Effectiveness of a physical activity intervention for head start preschoolers: a randomized intervention study. Am J Occup Ther. 2013;67(1): 28-36.

52. Chow BC, Chan L. Gross motor skills of Hong Kong preschool children. Asian J Phys Educ Recreat. 2011;17(1):71-7.

53. Goodway JD, Rudisill ME. Perceived physical competence and actual motor skill competence of African American preschool children. Adapt Phys Activ Q. 1997;14(4):314-26.

54. Iteya M, Gabbard C, Hart S. Limb laterality and motor proficiency in children. Int J Neurosci. 1995;83(3-4):275-9.

55. Lam HMY, Schiller W. A pilot study on the gross motor proficiency of Hong Kong preschoolers aged 5 to 6 years. Early Child Dev Care. 2001;171(1):11-20.

56. LeGear M, Greyling L, Sloan E, et al. A window of opportunity? Motor skills and perceptions of competence of children in kindergarten. Int J Behav Nutr Phys Act. 2012;9:29.

57. Morano M, Colella D, Caroli M. Gross motor skill performance in a sample of overweight and non-overweight preschool children. Int J Pediatr Obes. 2011;6(Suppl):42-6.

58. Nervik D, Martin K, Rundquist P, et al. The relationship between body mass index and gross motor development in children aged 3 to 5 years. Pediatr Phys Ther. 2011;23(2): $144-8$.

59. Olesen L, Kristensen P, Ried-Larsen M, et al. Physical activity and motor skills in children attending 43 preschools: a crosssectional study. BMC Pediatr. 2014;14(1):229.

60. Queiroz DdR, Ré AHN, Henrique RdS, et al. Participation in sports practice and motor competence in preschoolers. Motriz: Revista de Educação Física. 2014;20:26-32. 
61. Roberts D, Veneri D, Decker R, et al. Weight status and gross motor skill in kindergarten children. Pediatr Phys Ther. 2012;24(4):353-60.

62. Robinson LE. The relationship between perceived physical competence and fundamental motor skills in preschool children. Child Care Health Dev. 2010;37(4):589-96.

63. Robinson LE, Wadsworth DD, Peoples CM. Correlates of school-day physical activity in preschool students. Res Q Exerc Sport. 2012;83(1):20-6.

64. Saraiva L, Rodrigues LP, Cordovil R, et al. Influence of age, sex and somatic variables on the motor performance of pre-school children. Ann Hum Biol. 2013;40(5):444-50.

65. Temple VA, Crane JR, Brown A, et al. Recreational activities and motor skills of children in kindergarten. Phys Educ Sport Pedagogy. 2014. doi:10.1080/17408989.2014.924494.

66. Woodard RJ, Yun J. The performance of fundamental gross motor skills by children enrolled in head start. Early Child Dev Care. 2001;169(1):57-67.

67. Abbas J, Tedla JS, Krishnan S. Normative data for BruininksOseretsky Test of Motor Proficiency (BOTMP) in children of 91/2-141/2 years: a cross-sectional study. Crit Rev Phys Rehabil Med. 2011;23(1-4):125-33.

68. Bakhtiar S. Fundamental motor skill among 6-year-old children in Padang, West Sumatera, Indonesia. Asian Soc Sci. 2014;10(5):155-8.

69. Choi-Tse KC. A preliminary study on gross motor performance of Hong Kong children aged 6-8 years. J Phys Educ Recreat (Hong Kong). 2004;10(2):67-72.

70. Cohen KE, Morgan PJ, Plotnikoff RC, et al. Fundamental movement skills and physical activity among children living in low-income communities: a cross-sectional study. Int J Behav Nutr Phys Act. 2014;11(1):58-74.

71. D'Hondt E, Deforche B, De Bourdeaudhuij I, et al. Relationship between motor skill and body mass index in 5- to 10-year-old children. Adapt Phys Activ Q. 2009;26(1):21-37.

72. D'Hondt E, Deforche B, Gentier I, et al. A longitudinal analysis of gross motor coordination in overweight and obese children versus normal-weight peers. Int J Obes. 2013;37(1):61-7.

73. D'Hondt E, Deforche B, Gentier I, et al. A longitudinal study of gross motor coordination and weight status in children. Obesity. 2014;22(6):1505-11.

74. Habib Z, Westcott S, Valvano J. Assessment of balance abilities in Pakistani children: a cultural perspective. Pediatr Phys Ther. 1999;11(2):73-82.

75. Hume C, Okely A, Bagley S, et al. Does weight status influence associations between children's fundamental movement skills and physical activity? Res Q Exerc Sport. 2008;79(2):158-65.

76. Jones RA, Okely AD, Caputi P, et al. Relationships between child, parent and community characteristics and weight status among young children. Int J Pediatr Obes. 2010;5(3):256-64.

77. Junaid KA, Fellowes S. Gender differences in the attainment of motor skills on the Movement Assessment Battery for children. Phys Occup Ther Pediatr. 2006;26(1/2):5-11.

78. Kemp C, Pienaar AE. Relationship between the body composition and motor and physical competence of Grade 1 learners in South Africa. J Sports Med Phys Fit. 2013;53(6):635-43.

79. Larouche R, Boyer C, Tremblay MS, et al. Physical fitness, motor skill, and physical activity relationships in grade 4 to 6 children. Appl Physiol Nutr Metab. 2014;39(5):553-9.

80. Lopes L, Santos R, Pereira B, et al. Associations between sedentary behavior and motor coordination in children. Am J Hum Biol. 2012;24(6):746-52.

81. MacCobb S, Greene S, Nugent JK, et al. Measurement and prediction of motor proficiency in children using the Bayley Infant Scales and the Bruininks-Oseretsky Test. Phys Occup Ther Pediatr. 2005;25(1/2):59-79.
82. McKenzie TL, Sallis JF, Broyles SL, et al. Childhood movement skills: predictors of physical activity in Anglo American and Mexican American adolescents? Res Q Exerc Sport. 2002;73(3): 238-44.

83. McPhillips M, Jordan-Black J-A. The effect of social disadvantage on motor development in young children: a comparative study. J Child Psychol Psychiatry. 2007;48(12):1214-22.

84. Morrison KM, Bugge A, El-Naaman B, et al. Inter-relationships among physical activity, body fat, and motor performance in 6to 8-year-old Danish children. Pediatr Exerc Sci. 2012;24(2): 199-209.

85. Parvez F, Wasserman GA, Factor-Litvak P, et al. Arsenic exposure and motor function among children in Bangladesh. Environ Health Perspect. 2011;119(11):1665-70.

86. Ratzon N, Greenbaum C, Dulitzky M, et al. Comparison of the motor development of school-age children born to mothers with and without diabetes mellitus. Phys Occup Ther Pediatr. 2000;20(1):43-57.

87. Roeber BJ, Tober CL, Bolt DM, et al. Gross motor development in children adopted from orphanage settings. Dev Med Child Neurol. 2012;54(6):527-31.

88. Slotte S, Sääkslahti A, Metsämuuronen J, et al. Fundamental movement skill proficiency and body composition measured by dual energy X-ray absorptiometry in eight-year-old children. Early Child Dev Care. 2015;185(3):475-85. doi:10.1080/ 03004430.2014.936428.

89. Tsapakidou A, Anastasiadis T, Zikopoulou D, et al. Comparison of locomotor movement skills in 8-9 years old children coming from two areas of Thessaloniki with contrasting socioeconomic status. Asian J Humanit Soc Stud. 2014;2(1):186-92.

90. Vandendriessche JB, Vandorpe BFR, Vaeyens R, et al. Variation in sport participation, fitness and motor coordination with socioeconomic status among Flemish children. Pediatr Exerc Sci. 2012;24(1):113-28.

91. Vandorpe B, Vandendriessche J, Vaeyens R, et al. Relationship between sports participation and the level of motor coordination in childhood: a longitudinal approach. J Sci Med Sport. 2012;15(3):220-5.

92. Vedul-Kjelsås V, Stensdotter A-K, Sigmundsson H. Motor competence in 11-year-old boys and girls. Scand J Educ Res. 2013;57(5):561-70.

93. Venetsanou F, Kambas A. The effect of age and gender on balance skills in preschool children. FU Phys Ed Sport. 2011;9(1):81-90.

94. Wright MJ, Bos C. Performance of children on the community balance and mobility scale. Phys Occup Ther Pediatr. 2012;32(4):416-29.

95. Ziviani J, Poulsen A, Hansen C. Movement skills proficiency and physical activity: a case for Engaging and Coaching for Health (EACH)-Child. Aust Occup Ther J. 2009;56(4):259-65.

96. Barnett LM, Van Beurden E, Morgan PJ, et al. Gender differences in motor skill proficiency from childhood to adolescence: a longitudinal study. Res Q Exerc Sport. 2010;81(2):162-70.

97. Jaakkola $\mathrm{T}$, Washington $\mathrm{T}$. The relationship between fundamental movement skills and self-reported physical activity during Finnish junior high school. Phys Educ Sport Pedagogy. 2013;18(5):492-505.

98. Okely AD, Booth ML, Patterson JW. Relationship of cardiorespiratory endurance to fundamental movement skill proficiency among adolescents. Pediatr Exerc Sci. 2001;13(4):380-91.

99. Laukkanen A, Pesola A, Havu M, et al. Relationship between habitual physical activity and gross motor skills is multifaceted in 5- to 8-year-old children. Scand J Med Sci Sports. 2014;24(2):e102-10.

100. Okely AD, Booth ML, Chey T. Relationships between body composition and fundamental movement skills among children and adolescents. Res Q Exerc Sport. 2004;75(3):238-47. 
101. Woll A, Worth A, Mündermann A, et al. Age- and sex-dependent disparity in physical fitness between obese and normal weight children and adolescents. J Sports Med Phys Fit. 2013;53(1):48-55.

102. Ulrich DA. Test of gross motor development. 2nd ed. Austin: Pro-Ed; 2000.

103. Duncan M, Stanley M, Leddington Wright S. The association between functional movement and overweight and obesity in British primary school children. BMC Sports Sci Med Rehabil. 2013;5(1): 11 .

104. Hills AP, Hennig EM, Byrne NM, et al. The biomechanics of adiposity - structural and functional limitations of obesity and implications for movement. Obes Rev. 2002;3(1):35-43.

105. Butterfield SA, Angell RM, Mason CA. Age and sex differences in object control skills by children ages 5 to 14. Percept Mot Skills. 2012;114(1):261-74.

106. Blatchford P, Baines E, Pellegrini A. The social context of school playground games: sex and ethnic differences, and changes over time after entry to junior school. Br J Dev Psychol. 2003;21(4):481-505.

107. Eccles JS, Harold RD. Gender differences in sport involvement: applying the Eccles' expectancy-value model. J Appl Sport Psychol. 1991;3(1):7-35.

108. Hills LA, Croston A. 'It should be better all together': exploring strategies for 'undoing' gender in coeducational physical education. Sport Educ Soc. 2011;17(5):591-605.

109. Lee AM, Fredenburg K, Belcher D, et al. Gender differences in children's conceptions of competence and motivation in physical education. Sport Educ Soc. 1999;4(2):161-74.

110. Lee SM, Nihiser A, Strouse D, et al. Correlates of children and parents being physically active together. J Phys Act Health. 2010;7(6):776-83.

111. Wright J. The construction of gendered contexts in single sex and co-educational physical education lessons. Sport Educ Soc. 1997;2(1):55-72.

112. Bauman AE, Reis RS, Sallis JF, et al. Correlates of physical activity: why are some people physically active and others not? Lancet. 2012;380(9838):258-71.
113. Edwardson CL, Gorely T, Musson H, et al. Does activity-related social support differ by characteristics of the adolescent? J Phys Act Health. 2014;11(3):574-80.

114. Yao C, Rhodes R. Parental correlates in child and adolescent physical activity: a meta-analysis. Int J Behav Nutr Phys Act. 2015;12(1):10.

115. Fisher A, Reilly JJ, Kelly LA, et al. Fundamental movement skills and habitual physical activity in young children. Med Sci Sports Exerc. 2005;37(4):684-8.

116. Williams HG, Pfeiffer KA, O'Neill JR, et al. Motor skill performance and physical activity in preschool children. Obesity. 2008;16(6):1421-6.

117. Wrotniak BH, Epstein LH, Dorn JM, et al. The relationship between motor proficiency and physical activity in children. Pediatrics. 2006;118(6):e1758-65.

118. Haapala Eero A. Cardiorespiratory fitness and motor skills in relation to cognition and academic performance in children-a review. J Hum Kinet. 2013;36(1):55-68.

119. Jaakkola T, Hillman C, Kalaja S, et al. The associations among fundamental movement skills, self-reported physical activity and academic performance during junior high school in Finland. J Sports Sci. 2015:33(16):1719-29.

120. Myer GD, Faigenbaum AD, Edwards NM, et al. Sixty minutes of what? A developing brain perspective for activating children with an integrative exercise approach. Br J Sports Med. 2015. doi:10.1136/bjsports-2014-093661

121. Cools W, De Martelaer K, Samaey C, et al. Fundamental movement skill performance of preschool children in relation to family context. J Sports Sci. 2011;29(7):649-60.

122. Rudd J, Butson ML, Barnett ML, et al. A holistic measurement model of movement competency in children. J Sports Sci. 2016;34(5):477-85.

123. Vlahov E, Baghurst TM, Mwavita M. Preschool motor development predicting high school health-related physical fitness: a prospective study. Percept Mot Skills. 2014;119(1):279-91.

124. Cliff DP, Okely AD, Morgan PJ, et al. Movement skills and physical activity in obese children: randomized controlled trial. Med Sci Sports Exerc. 2011;43(1):90-100. 